\title{
Review Article \\ Oxidative Stress and Its Clinical Applications in Dementia
}

\author{
Peizhong Mao ${ }^{1,2}$ \\ ${ }^{1}$ The Division of Neuroscience, Oregon National Primate Research Center, Oregon Health \& Science University, \\ 505 NW 185th Avenue, Beaverton, OR 97006, USA \\ ${ }^{2}$ The Departments of Physiology and Pharmacology, Public Health and Preventive Medicine, Oregon Health \& Science University, \\ Portland, OR 97239, USA \\ Correspondence should be addressed to Peizhong Mao; maop@ohsu.edu
}

Received 5 June 2012; Accepted 16 July 2012

Academic Editor: Peter Crouch

Copyright (C) 2013 Peizhong Mao. This is an open access article distributed under the Creative Commons Attribution License, which permits unrestricted use, distribution, and reproduction in any medium, provided the original work is properly cited.

\begin{abstract}
Dementia is a complex disorder that mostly affects the elderly and represents a significant and growing public health burden in the world. Alzheimer's disease (AD)- associated dementia and dementia with Lewy bodies (DLB) are the most common forms of dementia, in which oxidative stress is significantly involved. Oxidative stress mechanisms may have clinical applications, that is, providing information for potential biomarkers. Thus brain-rich peptides with an antioxidant property, such as CART (cocaineand amphetamine-regulated transcript), may be promising new markers. This paper summarizes the progress in research regarding oxidative stress in dementia with a focus on potential biomarkers in the cerebrospinal fluid (CSF) in the main forms of dementia. Other central and peripheral biomarkers, especially those considered oxidative stress related, are also discussed. This paper aims to provide information to improve current understanding of the pathogenesis and progression of dementia. It also offers insight into the differential diagnosis of AD and DLB.
\end{abstract}

\section{Introduction}

Dementia is a multisystem-related neurodegenerative disorder. According to the DSM-IIIR (the Diagnostic and Statistical Manual, 3rd edition, revised) the essential feature of dementia is impairment in short- and long-term memory, associated with impairment in abstract thinking, impaired judgment, other disturbances of higher cortical function, or personality change. The disturbance is severe enough to interfere significantly with work or usual social activities or relationships with others. The diagnosis of dementia is not made if these symptoms occur in delirium. The DSM-IIIR definition of dementia is reliable and is routinely used in clinical guidelines $[1,2]$.

There are several forms of dementia, including dementia associated with Alzheimer's disease (AD), dementia with Lewy bodies (DLB), corticobasal degeneration/dementia (CBD), frontotemporal dementias (FTD) (also known as frontotemporal lobar degenerations or FTLD), vascular dementia (VAD), and prion diseases such as CreutzfeldtJakob Disease (CJD) [1, 3]. Among all forms of dementia,
Alzheimer's dementia and dementia with Lewy bodies are the most common.

$\mathrm{AD}$ is accounting for $60-80 \%$ of the total number of dementia, characterized by extracellular fibrillar amyloid $\beta$ $(\mathrm{A} \beta)$, especially long form 42 amino acids of $\mathrm{A} \beta(\mathrm{A} \beta 42)$ deposits (amyloid plaques), intracellular neurofibrillary tangles (NFT, phosphate-tau related), and neuronal as well as axonal degeneration in the brain [4-6].

Dementia with Lewy bodies (DLB), accounting for $15-30 \%$ of the total number of recorded cases of dementia, is the second most common form of dementia and is characterized by a dysexecutive-visuoperceptual dementia frequently accompanied by visual hallucinations, fluctuating attention, and Parkinsonism. Strategic $\alpha$-synuclein ( $\alpha$ Syn) aggregates, neuronal loss, and variable degrees of amyloid deposition constitute the key pathological features [1, 7-9].

Importantly, mitochondria ( $\mathrm{mt})$, the powerhouse in most cells, are pivotal in controlling cell survival and death. Cumulative oxidative stress, disrupted mitochondrial respiration, and mitochondrial damage are involved in various neurodegenerative disorders, including AD and DLB [4-6]. 
The neuropeptide CART is expressed in the nervous system and circulatory system with multiple functions. We recently found that CART is a mitochondrial booster and a novel endogenous antioxidant $[10,11]$, indicating its new utility in neurodegenerative diseases. For example, CART may be a disease biomarker and/or therapeutic target.

Increasing evidence indicates the pathogenic role of oxidative stress in the early phase of neurodegeneration. As such, oxidative stress mechanisms may suggest novel disease markers. In this paper, I attempt to summarize the role of oxidative stress in dementia, with a focus on the cerebrospinal fluid (CSF) biomarkers in $\mathrm{AD}$ and DLB, including their relationship with CART peptide.

\section{Oxidative Stress in the Etiopathology of Dementia}

2.1. Oxidative Stress in Alzheimer's Dementia. A growing body of evidence suggests that $\mathrm{AD}$ is a multifactorial and the most common, neurodegenerative disease. Senile plaques (A $\beta$ plaques) and neurofibrillary tangles (tau pathology) are the hallmarks in its pathology. AD is a progressive disease and AD-related pathophysiological changes including oxidative damage can occur many years and even decades before the appearance of clinical dementia syndrome. Therefore, the latest criteria proposed last year for the neuropathologic assessment of AD differ from the traditional criteria [2]. These criteria describe three defined stages in a clinical continuum that includes preclinical, mild cognitive impairment, and dementia [2].

Recent progress in $\mathrm{AD}$, especially $\mathrm{A} \beta$, mitochondria, and oxidative stress-related pathology has been reviewed $[6$, $12,13]$. Briefly, $\mathrm{A} \beta$ (especially $\mathrm{A} \beta 42$ ) can damage neurons by directly inducing production of reactive oxygen species (ROS), indicating A $\beta$ peptides are probably oxidant peptides. ROS are mainly generated in mitochondria, in particular from mitochondrial dysfunction. In other words, mitochondria may have an etiological role in the development of AD. Excessive ROS damage the components of the cell, including mitochondrial DNA (mtDNA), nuclear DNA, RNA, proteins, and lipids and eventually result in synapse damage and neuronal cell death. When this occurs in the memory center, the hippocampus, and associated key brain areas, it produces the clinical manifestations of AD.

Importantly, oxidative damage may possibly be the earliest event in $\mathrm{AD}$, based on postmortem studies showing that increases in neuronal $80 \mathrm{HdG}$ (8-hydroxy-2' deoxyguanosine) and 3-nitrotyrosine, which are markers of DNA and protein oxidation, respectively, precede $\mathrm{A} \beta$ plaques and NFTs $[6,14,15]$. There is approximately a twofold increase in DNA damage in lymphocytes of patients with mild cognitive impairment (MCI; a strong risk for developing $\mathrm{AD})$ and $\mathrm{AD}[6,16]$. Using a redox proteomics approach, several specific targets of protein oxidation such as peptidyl prolyl cis-trans isomerase and ubiquitin carboxyl terminal hydrolase 1 have been identified in the hippocampus but not in the cerebellum in $\mathrm{AD}$ [17], indicating that oxidatively modified proteins were consistent with biochemical or pathological alterations in AD. Furthermore, some peripheral markers of oxidative stress such as high malonaldehyde (MDA) levels (representing lipid peroxidation), low glutathione levels, and low glutathione reductase appear in MCI with a similar pattern to that observed in AD. Taken together, these findings suggest that oxidative stress may represent a signal of AD pathology, even an early signal in very mild cognitive disorders. Therefore, AD and MCI are biochemically equivalent [18-20]. Not surprisingly, increased protein and lipid oxidative damage was also found in AD brain tissue and mitochondria isolated from lymphocytes of $\mathrm{AD}$ patients, further indicating the role of oxidative stress in $\mathrm{AD}$ etiopathology $[21,22]$.

Toxic soluble $\mathrm{A} \beta$ oligomers appear to have synaptic receptors colocalizing with PSD-95 (postsynaptic density protein 95), and $\mathrm{A} \beta 42$ accumulates in dendrites in $\mathrm{AD}$ patients where it may cause oxidative damage, caspase activation, and finally apoptosis [23]. Docosahexaenoic acid (DHA), an essential omega-3 polyunsaturated fatty acid (PUFA), is a major component of neuronal membrane phospholipids. It is enriched in synapses and central to postsynaptic signaling and neuroprotection. It has been shown that DHA serum level is reduced in $\mathrm{AD}$ patients [24]. Further, the neuronal membranes of AD patients have been found to be deficient in DHA $[25,26]$. The loss of DHA in $\mathrm{AD}$ may reflect its propensity for free radicalmediated lipid peroxidation, resulting in its conversion to neuroprostanes (F4-isoprostanes), which are elevated in $\mathrm{AD}$ [27, 28]. F2-isoprostanes (F2-IsoPs) are other indicators of lipid peroxidation, which are exclusive products of free radical-mediated damage to arachidonic acid and released into extracellular fluid. F(2)-IsoPs are increased in diseased regions of the brain from definite $\mathrm{AD}$ patients compared to controls [29]. On the other hand, a diet enriched with the omega-3 fatty acid DHA reduces amyloid burden in Alzheimer's mouse models [30, 31].

In addition, aging is thought to be the single most important risk factor for $\mathrm{AD}$, and aging invariably decreases sensory stimuli and impacts on the thalamocortical system and its connectivity to key regions of the brain. Memory dysfunction in senescence and early $\mathrm{AD}$, a function of acetylcholine decrease, is accompanied by dysfunctional basal forebrain, parietal, prefrontal, and entorhinal cortices, and indeed the hippocampus [32-34]. Cholinergic neurotransmission protects neurons from $\mathrm{A} \beta$ production and its toxicity, while cholinergic depletion enhances both [34]. Increasing data indicate that memory disturbances in normal elderly and early $\mathrm{AD}$ patients are intimately related to hypoxia, a reduction in blood supply, and glucose hypometabolism in the hippocampus and a number of key brain areas. The recent hypothesis on memory impairment in the elderly and Alzheimer's dementia, therefore, underscores age-related sensory losses, functional disconnection between strategic brain regions in conjunction with hypoxemia, and hypometabolism [34]. Notably, the hypoxia condition can increase $\mathrm{A} \beta$ production via the limiting enzyme $\beta$-site APP-cleaving enzyme 1 (BACE1) and gamma secretase. Hypoxia also stimulates 
ROS production, especially the radicals produced from mitochondrial complex II $[6,35,36]$. Very recently the first direct evidence in living humans that ischemia acutely increases $A \beta 42$ levels has been reported [37].

Further, decreased cerebral microperfusion may be partially due to altered metabolism of nitric oxide (NO), which mediates the effect of endothelium-derived relaxing factor in blood vessels and plays a part in neuronal communication in the brain. The NO synthase (NOS) is expressed in the brain and regulated by many different factors [38, 39]. Human brain microvascular endothelial cell experiments showed that inhibition of endothelial NOS (eNOS) with the specific NOS inhibitor L-NAME $(\mathrm{N}(\mathrm{G})$-nitro-l-arginine methyl ester) led to increased APP and BACE1 protein levels, as well as increased secretion of $\mathrm{A} \beta 42(P<0.001)$. Animal experiments showed brain tissue from eNOS (-/-) mice had statistically higher APP and BACE1 protein levels, as well as increased BACE1 enzyme activity and $\mathrm{A} \beta$ level [40]. These data suggest that endothelial NO plays an important role in modulating APP expression and processing within the brain and cerebrovasculature and that a possible molecular link between cardiovascular risk factors and AD identified as endothelial dysfunction is specifically decreased bioavailability of nitric oxide.

Interestingly, asymmetrical dimethylarginine (ADMA) is an endogenous inhibitor of NOS and may alter NO production during pathological conditions. It has been found that ADMA plasma concentrations were increased, while CSF ADMA concentrations were decreased in AD patients, and there was a significant association between decreasing CSF ADMA levels and the severity of cognitive impairment [41]. Decreased levels of CSF ADMA might lead to a cerebral increase of NO, peroxynitrite production, and oxidative protein damage, while elevated ADMA in plasma might be a contributing factor for $\mathrm{AD}$ through alterations of $\mathrm{NO}$ metabolism and decreased cerebral microperfusion.

Maintaining iron homeostasis is a critical issue for normal cell and brain functions since iron is an essential component of proteins containing iron-sulfur clusters, heme, and diironoxo metal centers that are involved in important cellular processes like oxygen transport, mitochondrial respiration, or DNA synthesis. Iron dyshomeostasis may be a causative factor for $\mathrm{AD}$ and other neurodegenerative disorders [4244]. In living organisms, iron can be found in its reduced ferrous $\left(\mathrm{Fe}^{2+}\right)$ and its oxidized ferric $\left(\mathrm{Fe}^{3+}\right)$ states. Severe iron deficiency will cause growth arrest and cell death, while excess free iron is involved in the Fenton redox reaction, which catalyzes the conversion of ROS to the highly reactive hydroxyl radical; further APP expression is regulated by iron $[43,45]$. Iron accumulation was observed in the brain regions (such as hippocampus) that are affected by $\mathrm{A} \beta$ deposition and neurons that contain neurofibrillary tangles in $\mathrm{AD}$ patients [43]. Recently, increased iron and free radicals have been found in both the cortex and the cerebellum of preclinical AD and MCI cases [46].

Overall, in the majority of $\mathrm{AD}$, dysfunction in mitochondria and metabolism produces excessive free radicals (ROS/RNS) and $\mathrm{A} \beta$ that damage essential cellular components such as nucleic acids (DNA/RNA), lipids, and proteins in the brain; oxidative damage is one of the earliest events in the development of $\mathrm{AD}$, including its preclinical stage and at the onset of mild cognitive impairment (Figure 1). This feature also suggests that $\mathrm{MCI}$ is the same as $\mathrm{AD}$ in the biochemistry-related pathological mechanisms, in other words, mitochondria/oxidative stress hypothesis of $\mathrm{AD}$ applies mostly to, if not all cases, but at least the common, late-onset of $\mathrm{AD}$. Hence, in the clinic, treatment of $\mathrm{AD}$ should be started at its very early stages, and mitochondria improvements should benefit the most $\mathrm{AD}$ patients.

\subsection{Antioxidant Defense System in Alzheimer's Dementia.} Importantly, in mammalian cells, individuals have a powerful antioxidant system against free radicals/ROS, including small molecules and antioxidant enzymes. There is a defect in the antioxidant defense system, however, which may lead to oxidative damage in patients with AD. Glutathione (GSH) is the most abundant and important antioxidant in our bodies. Noninvasive magnetic resonance spectroscopy has shown that healthy young male and female subjects have higher amount of GSH in the parietal cortical region and a specific GSH distribution pattern (parietal cortex $>$ frontal cortex $>$ hippocampus $\sim$ cerebellum), as well as higher amounts of GSH in the frontal cortical region compared to AD patients [47]. It has been found that erythrocyte antioxidant enzyme activities (catalase CAT, glutathione peroxidase GPX, and superoxide dismutase SOD) were significantly lower in patients with $\mathrm{AD}$ compared with controls [48]. Further more, glutathione reductase (GR) activity was lower in both MCI and $\mathrm{AD}$ patients than in aged subjects in relatively good health. Cognitive performance evaluated by the Mini-Mental State Examination (MMSE) was negatively associated with lipid peroxidation MDA levels $(r=-0.31, P=0.028)$ and positively correlated with GR/GPX ratio in AD patients ( $r=$ $0.68, P<0.001)[20]$. These results suggest that alterations in these enzymes may play a role in the etiopathogenesis of AD. The expression of key oxidative stress-mitigating genes in different brain regions in $\mathrm{AD}$ has also been investigated using reverse transcriptase-polymerase chain reaction (RT-PCR) [49]. The levels of peroxidation mitigating (normalized CAT, GPX, and GR) mRNAs were elevated in the hippocampus and inferior parietal lobule, but not in the cerebellum of $\mathrm{AD}$ patients, which may reflect the protective gene response to the increased peroxidation in the brain regions showing severe AD pathology [49].

Decreased levels of polyunsaturated fatty acids and increased levels of markers of lipid peroxidation have been reported in the brain in $\mathrm{AD}$, particularly in areas severely affected in the disease. 4-Hydroxynonenal (HNE), one marker of lipid peroxidation, is neurotoxic and elevated in $\mathrm{AD}$ brain and CSF. Furthermore, glutathione S-transferase (GST), a protective enzyme against aldehydes, and especially HNE, has been investigated in multiple brain regions in short-postmortem-interval AD patients [50]. A decrease in GST activity in all brain areas was observed in AD compared with age-matched controls and particularly in the amygdala, hippocampus, and parahippocampal gyrus. Expression of 


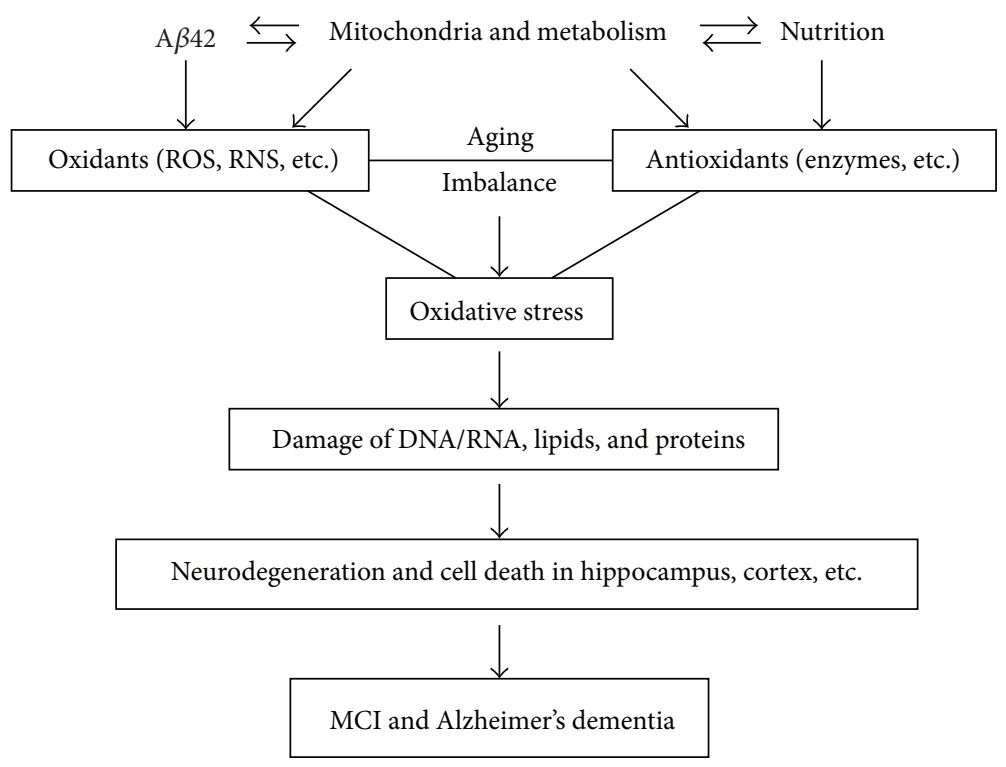

Figure 1: Oxidative stress in MCI and Alzheimer's dementia.

GST protein was also depleted in most regions of the brain in AD. These data indicate that reduced levels of the antioxidant enzyme GST have a role in the pathogenesis of neuron degeneration in $\mathrm{AD}$ [50].

Biliverdin reductase-A (BVR-A) is another antioxidant enzyme and drug target, together with heme oxygenase (HO); BVR-A forms a powerful system involved in the cell stress response during neurodegenerative disorders including $\mathrm{AD}$. It has been shown that BVR-A undergoes posttranslational oxidative and nitrosative modifications in the hippocampus, but not the cerebellum, of subjects with $\mathrm{AD}$ and amnestic MCI [51, 52]. A significant increase of nitrated BVR-A was demonstrated only in AD and MCI hippocampi, whereas no significant modifications were found in cerebellar tissues. These results supported the hypothesis of a prevalence of nitrosative stress-induced modifications on BVR-A structure, and this evidence was confirmed by a significant upregulation of inducible nitric oxide synthase in hippocampal tissue of subjects with $\mathrm{AD}$ and MCI that was not present in cerebellum [51]. Therefore, nitrosative stress-induced modifications on hippocampal BVR-A are an early event in the pathogenesis of $\mathrm{AD}$. In addition, HO-1 protein levels were significantly increased in the hippocampus of AD subjects, whereas HO2 protein levels were significantly decreased in both $\mathrm{AD}$ and MCI hippocampi. Further, significant increases in Serresidue phosphorylation together with increased oxidative posttranslational modifications were found in the hippocampus of $\mathrm{AD}$ subjects [53]. These data indicate that different forms of antioxidant enzymes may have different responses and roles to the oxidative stress in the brain.

Oxidatively damaged molecules need to be removed or repaired. The ubiquitin-proteasome mechanism is involved in this process [54-56]. In Alzheimer's disease, there is a 2.5 -fold increase in the total endosome volume in cortical neurons suggesting increased endocytic activity [57-59]. This increased rate of endocytosis in $\mathrm{AD}$ may be in response to the $\mathrm{A} \beta$ protein load or to the need to repair the damaged proteins in the brain, or both. It has been demonstrated that proteasomes are concerned with the removal of oxidized proteins, which plays an essential role in the cellular defense against oxidative attack [60].

To date, successful antioxidant therapy for $\mathrm{AD}$ has not been obtained. One reason is the difficulty of the most antioxidants to pass the blood-brain barrier. To overcome this problem, recently we and other research groups found that mitochondria-targeted catalase (MCAT) has a beneficial role in both transgenic AD model mice and wild-type mice through multiple mechanisms including reduction of ROS and oxidative damage, as well as toxic $\mathrm{A} \beta 42$ production and deposits in the brain $[61,62]$, further indicating that mitochondria and oxidative stress play an important role in $\mathrm{AD}$ and normal aging.

Because increased oxidative damage and reduced antioxidant enzymes in the hippocampus and cortex are significantly involved in the development of AD (summarized in Figure 1); pathology-related local and global biomarkers in the individuals may help to detect the preclinical stages of disease and improve early and differential diagnosis. Core $\mathrm{AD}$ biomarkers, $\mathrm{A} \beta$ peptides (especially $\mathrm{A} \beta 42$ isoform) and phosphor-tau (p-tau) in cerebrospinal fluid (CSF), PET, and MRI are considered to be the most useful approaches in terms of diagnostic and predictive value in preclinical and clinical stages of $\mathrm{AD}[6,63-68]$. Importantly, a reduced concentration of $\mathrm{A} \beta 1-42$ in CSF may provide the earliest definitive evidence of Alzheimer's pathology in the brain since some individuals who have low concentrations of CSF A $\beta 1-42$ show no evidence of amyloid on PET PIB scans. However, autopsy may show widespread diffuse-but minimal fibrillar-amyloid-plaque deposits [69]. Because CSF is the most cost-effective and reliable approach diagnostic, it is the focus of this paper. 
2.3. Oxidative Stress in Dementia with Lewy Bodies. DLB has been defined clinically by the presence of dementia, gait/balance disorder, prominent hallucinations and delusions, sensitivity to traditional antipsychotics, and fluctuations in alertness [70]. These diagnostic guidelines have been confirmed by recent workshops [2, 71].

In pathology, DLB is one of the Lewy body diseases, so DLB shares some features of Parkinson's disease (PD). Filamentous protein inclusions in neurons (Lewy bodies, LB) and dystrophic neuritis (Lewy neuritis, LN) containing pathologic $\alpha$-synuclein ( $\alpha$ Syn) are the morphologic hallmarks of dementia with Lewy bodies and sporadic PD. They occur in the central, peripheral, and autonomic nervous system as essential or coincidental features. Their formation runs through several phases from initial dust-like particles cross-linked with $\alpha$ Syn to aggregation of ubiquitinated dense filaments, leading to the formation of LBs, in which $\alpha$ Syn is the major component and hundreds of other proteins may be included [72], to the final degradation and death of the afflicted neurons [8]. The disease progression-related decreases of dopamine, dopamine transporter (DAT), and tyrosine hydroxylase $(\mathrm{TH})$ expression in striatum are negatively correlated with total substantia nigra (SN) $\alpha$ Syn burden and neuronal loss $[4,73]$. Glutathione is the most abundant antioxidant involved. The reduced form (GSH(R)), but not oxidized glutathione (GSSG), has been shown to be dramatically altered in the SN of Lewy body disease patients post mortem; similarly, reduced but not oxidized glutathione levels in cerebrospinal fluid are lowered in Lewy body diseases therefore; it has been thought that the alterations of the glutathione system occur in a very early stage of the disease or may even represent a risk marker for LBD [74]. These pathological changes are important targets for disease diagnosis and therapy. For example, reduced DAT levels in DLB as shown with [123I]FP-CIT-SPECT is recognized as the most reliable and valid biomarker $[75,76]$.

Oxidative damage, particularly lipoxidation, advanced glycation (AGE), and AGE receptors (RAGE) in the cerebral cortex have also been seen in early stages of diseases with Lewy bodies [77]. Moreover, it demonstrates that $\alpha$ Syn lipoxidation is an early event in LBDs that precedes $\alpha$ Syn solubility modification, aggregation, and formation of Lewy bodies and neuritis [78]. It has been found that in human brain cortex, mitochondrial oxygen uptake, and complex I activity were significantly lower in PD and DLB, compared to healthy controls, whereas oxidative damage and mtNOS activity, cytochrome content, expression of Mn-SOD, and mitochondrial mass were significantly higher in the frontal cortex in PD and DLB [79]. The decreases in tissue and mitochondrial oxygen uptake and in complex I activity are considered the consequences of mitochondrial oxidative damage and mitochondrial dysfunction. The increases in mtNOS activity and in mitochondrial mass are interpreted as an adaptive response of the frontal cortex that involves increased NO signaling for mitochondrial biogenesis. The adaptive response would partially compensate for mitochondrial dysfunction in these neurodegenerative diseases and would afford a human evolutionary response to shortage of
ATP in the frontal cortex. This compensatory mechanism also occurs in aged nonhuman primates [80].

Interestingly, shortened telomere length is associated with various age-related diseases, especially in dementia such as AD. Similar changes in telomere length in dementia with DLB have also been reported recently. Telomere length was significantly shorter in the DLB group than in the nondemented elderly control (NEC) group. Urinary 8-OHdG levels were significantly higher in the DLB group than in the NEC group. There was a negative correlation between telomere length and age in the DLB group [81].

The relationship between telomere length and oxidative stress has been investigated in several other diseases [8285]. For example, telomere length was inversely correlated with oxidative stress in subjects with clinical depression [86]. Shorter leukocyte telomere length (LTL) is also associated with the presence of T2D and this could be partially attributed to the high oxidative stress in these patients [87]. In healthy women it has been shown that LTL is associated with cognitive performance [88], and decreasing telomere length was strongly correlated with decreasing hippocampal volume [89]. These preliminary data suggest that telomere length may be a possible early marker of DLB and other dementia risk.

Heavy metals have been implicated as the causative agents for the pathogenesis of the most prevalent neurodegenerative diseases. Various mechanisms have been proposed to explain the toxic effects of metals ranging from metal-induced oxidation of protein to metal-induced changes in the protein conformation. Aggregation of $\alpha$-synuclein is a key mechanism in Parkinson's disease and DLB, and various metals, including copper, constitute a prominent group of alphasynuclein aggregation enhancers. Using a set of biophysical techniques, the $\alpha$ Syn-Cu21 binding sites were systematically characterized and analyzed for the possible role of metal binding in $\alpha$ Syn fibrillation. The analyses indicated that $\alpha$ Syn possesses at least two binding sites for $\mathrm{Cu} 21$. One of these binding sites is found in the N-terminal region [90].

On the other hand, lipoxidative protein damage of aldolase A, enolase 1, and glyceraldehyde dehydrogenase (GAPDH) was found in the frontal cortex in the majority of cases of incidental Parkinson's disease (iPD), PD, and dementia with LB. Densitometric studies have shown that the ratio of oxidized protein per spot is higher in $\mathrm{PD}$, PD, and DLB compared with controls. These findings show oxidation of three enzymes linked with glycolysis and energy metabolism in the adult human brain as well as increased oxidation of aldolase A, enolase 1, and GAPDH in the frontal cortex in Lewy body diseases [91].

In mammalian cells, glutathione peroxidase (GPX-1) is one of the main antioxidant enzymes inactivating hydrogen peroxide and protecting against oxidative stress. It has been found that the highest levels of GPX-1 occur in microglia with lower levels in neurons. Unstructured Lewy bodies were enveloped with a layer of GPX-1 that was partially colocalized with alpha-synuclein whereas concentric Lewy bodies had discrete deposits of GPX-1 around the periphery, which appeared to be involved in the degradation of the Lewy bodies. Abnormal alpha-synuclein as found in Lewy bodies 
produces hydrogen peroxide and these neurons are capable of directing antioxidant enzymes to regions of oxidative stress [92]. On the other hand, this endogenous defense may reflect a pathological mechanism and therapeutic target.

Taken together, these findings support the idea that oxidative stress is involved in the pathological process of neurodegenerative diseases and antioxidant therapy in the treatment of DLB and AD is reasonable to reduce oxidative stress-associated neuronal damage [61, 93]. Further, oxidative stress-related biomarkers might have the potential to assist in the diagnosis of neurodegenerative diseases.

\section{CSF Biochemical Markers in AD and DLB}

\subsection{Core CSF Biomarkers and Oxidative Stress Markers in AD} and MCI. Physiological processes and pathological changes in the brain can be monitored by analysis of cerebrospinal fluid (CSF). CSF markers reflect neuropathology in $94 \%$ of $\mathrm{AD}$-associated cases of dementia [3]. As discussed above, $\mathrm{A} \beta$ depositions, neurofibrillary tangles, and oxidative damage are the main pathological features of $\mathrm{AD}$. These featurerelated biochemical changes in CSF are important signals for the disease initiation and development. For the past two decades, there has been intense interest in developing markers related to the neuropathology of AD in CSF. Several key CSF biomarkers of pathological processes in the brain are now available. CSF levels of 42-amino acid isoform of amyloid $\beta$ (A $\beta 42)$, which directly induces reactive oxygen species, reflect brain amyloid pathology; levels of total tau reflect cortical axonal degeneration and levels of phosphotau reflect tangle pathology [94]. While toxic A $\beta 42$ forms and deposits plaques in the brain, the A $\beta 42$ level in CSF is decreased accordingly. Most reports focused on these $\mathrm{A} \beta$ peptides and tau, showing mainly CSF A $\beta 42$ is reduced but CSF $\mathrm{A} \beta 40$ and tau are increased in $\mathrm{AD}$ patients as well $[1,63]$.

Aging and amyloid $\beta$ can induce oxidative stress and oxidative damage in the brain is considered the earliest event in AD. Accordingly, it is clear that CSF levels of $\mathrm{A} \beta 42$ retain diagnostic utility in patients with very mild $\mathrm{AD}$ and even mild cognitive impairment, and a combination of CSF t-tau and A $\beta 42$ at baseline yielded a sensitivity of $~ 95 \%$ and a specificity of $\sim 83 \%$ for detection of incipient AD in patients with MCI [95-98]. These data have been confirmed by a recent large-scale multicenter investigation [99-101]. It has been shown that cutoffs with sensitivity set to $85 \%$ were defined in the $\mathrm{AD}$ control groups and tested in the MCI group, where the combination of $\mathrm{A} \beta 42 / \mathrm{p}$-tau ratio and $\mathrm{t}-$ tau identified incipient AD with a sensitivity of $83 \%(95 \%$ CI, $78 \%-88 \%)$ and specificity of $72 \%$ (95\% CI, $68 \%-76 \%)$ [100]. This identification of incipient AD with good accuracy, though less accurately than previous single-center studies, indicates a need for standardization of analytical techniques and clinical procedures, including the improvement of the analytical kits, for large-scale or multicenter studies [102104]. Interestingly, cued recall deficits are most closely associated with CSF biomarkers indicative of $\mathrm{AD}(\mathrm{A} \beta 42 /$ tau ratio, CSF AD+ group) in subjects with $\mathrm{MCI}[105,106]$.
Though the diagnostic accuracies for AD decreased with age, the predictive values for a combination of biomarkers remained essentially stable, including the use of CSF biomarkers for $\mathrm{AD}$, even in older populations [107]. The Alzheimer's Disease Neuroimaging Initiative (ADNI) has shown that diagnostic threshold CSF concentrations for $\mathrm{A} \beta 42$ and for the ratio $\mathrm{t}$-tau/A $\beta 42$ were determined in an ADNI-independent autopsy-confirmed AD cohort from whom antemortem CSF was obtained, and a clinically defined group of cognitively normal controls provides statistically significant separation of those who progressed from MCI to $\mathrm{AD}$ in the ADNI study. These data suggest that interrogation of antemortem CSF in cognitively impaired individuals to determine levels of $\mathrm{t}$-tau, $\mathrm{p}$-tau, and $\mathrm{A} \beta 42$, together with MRI and amyloid imaging biomarkers, could replace autopsy confirmation of AD plaque and tangle pathology as the "gold standard" for the diagnosis of definite $\mathrm{AD}$ in the near future [101].

Isoprostane is an important oxidative stress biomarker and it may be one of the earliest markers for neuronal damage related to $\mathrm{AD}$ [108]. It has been shown that cerebrospinal fluid lipoproteins are more vulnerable to oxidation in AD and cerebrospinal fluid levels of F2-IsoPs are elevated in probable AD patients compared to age-matched controls [109-111]. Furthermore, combined analysis of CSF Ab42 and F2-IsoP levels largely preserved sensitivity and improved specificity relative to classification with $\mathrm{A} \beta 42$ and tau alone, indicating its utility as an AD biomarker [112]. Analysis of CSF F2- IsoPs in 421 clinically normal individuals has shown a significant increase in CSF F2- IsoPs over the adult human lifespan $(P<$ 0.001) [113], indicating a correlative relationship between free radical injury in the central nervous system and aging. In addition, increased CSF F2-IsoPs levels were present in clinically normal subjects with the biomarker signature of $\mathrm{AD}(P<0.05)$ and those subjects with increased CSF tau $(P<0.001)$ [113]. Elevated F2-IsoP levels in the CSF were also found in preclinical familial AD (FAD) mutation carriers [114]. Interestingly, isoprostane may be a predictive parameter for the development of AD. Cognitively normal (NL) individuals with a maternal history $(\mathrm{MH})$ of late-onset Alzheimer's disease (LOAD) showed higher IsoP and reduced A $\beta(42 / 40)$ CSF levels compared with NL with a paternal history $(\mathrm{PH})$ and NL with a negative family history of LOAD $(\mathrm{NH})$ ( $P$ values $\leq .05)$, whereas no differences were found between $\mathrm{NH}$ and $\mathrm{PH}$. No group differences were found for $\mathrm{p}$-tau(231) and t-tau. The IsoP and $\mathrm{A} \beta(42 / 40)$ levels were correlated only within the MH group $\left(R^{2}=.32, P=.005\right)$. Therefore, adult children of LOAD-affected mothers express a pathobiological phenotype characterized by $\mathrm{A} \beta$-associated oxidative stress consistent with $\mathrm{AD}$, which might reflect increased risk for developing the disease [115] (Table 1).

Very interestingly, some markers of oxidative damage are also elevated in mitochondria isolated from $\mathrm{AD}$ lymphocytes. This not only provides insight into the important role of oxidative stress and mitochondria in $\mathrm{AD}$ development but also indicates that these oxidative stress indices potentially could serve as a viable biomarker for this dementing disorder [22]. 
A novel misfolded protein assay for the detection of soluble oligomers composed of $A \beta x-40$ and $x-42$ peptides from CSF has also been developed. Preliminary validation of this assay with 36 clinical samples demonstrated the presence of aggregated $\mathrm{A} \beta \mathrm{x}-40$ in the CSF of $\mathrm{AD}$ patients. Together with measurements of total $\mathrm{A} \beta 42$, diagnostic sensitivity and specificity greater than $95 \%$ and $90 \%$, respectively, were achieved. Although larger sample populations will be needed to confirm this diagnostic sensitivity, these studies demonstrate a sensitive method of detecting circulating $\mathrm{A} \beta \mathrm{x}$ 40 oligomers from $\mathrm{AD}$ CSF and suggest that these oligomers could be a powerful new biomarker for the early detection of $\mathrm{AD}[116]$.

Mitochondrial coenzyme Q-10 (CoQ) is an antioxidant that receives electrons from complex I and II. It has been shown that the percentage of oxidized/total CoQ (\%CoQ) in the CSF of the AD group $(78.2 \pm 18.8 \%)$ was significantly higher than in the control group $(41.3 \pm 10.4 \%)(P<0.0001)$. The concentration of 8-OHdG in the CSF of AD patients was greater than in the CSF of controls $(P<0.0001)$ and was positively correlated with the duration of illness $(r(s)=0.95, P<0.0001)$. The \%CoQ was correlated with concentrations of 8-OHdG in the CSF of AD patients $(r(s)=$ $0.66, P<0.001)$, further suggesting both mitochondrial oxidative damage and oxidative DNA damage play important roles in the pathogenesis of early $\mathrm{AD}$ development, and $\mathrm{CoQ}$ may be a useful biomarker for the disease [117].

As described above, there is a significant decrease in antioxidant GST activity and protein expression in multiple brain regions, especially the hippocampus in shortpostmortem-interval AD patients [50]. Similarly, a significant decrease in GST activity and protein levels was also found in ventricular CSF in AD [50], indicating that reduced levels of antioxidant enzyme GST may have an important role in the pathogenesis of $\mathrm{AD}$ and may serve as a CSF biomarker for this degenerative disease.

Overall, the primary focus of $\mathrm{AD}$-associated study has been directed toward amyloid and tau pathology and their relations to synaptic and neuronal loss. However, as the complexity of the disease becomes increasingly evident, the importance of other factors such as oxidative stress and mitochondrial dysfunction becomes apparent. Therefore, in addition to the core biomarkers (reduced $\mathrm{A} \beta 42$ and increased tau in AD CSF), oxidative stress-related biomarkers may also have potential utility in diagnosis of $\mathrm{AD}$, including preclinical and very early-stage disease. The principal $\mathrm{AD}$ markers are listed in Table 1.

3.2. Oxidative CSF Biomarkers in DLB. The clinical diagnosis of dementia with Lewy bodies (DLB) is made on the basis of consensus criteria; however, the sensitivity of the criteria is relatively low [9]. Differentiating dementia with Lewy bodies from $\mathrm{AD}$ can be difficult because of the substantial overlap in clinical features. A classification of patients with $\mathrm{AD}$ and patients with other dementias accomplished by combination of CSF $A \beta 42$ and $p$-tau shows that the CSF AD biomarker profile was seen in $47 \%$ of patients with DLB [3]. However, from many reports, a conclusive proposal, that is, a combination of CSF measures appears to emerge, that may well be able to differentiate DLB from other dementias: $\alpha$-synuclein reduction in early DLB, a correlation between CSF $\alpha$-synuclein and A $\beta 42$ measures (characteristic for DLB only), and t-tau and $\mathrm{p}$-tau181 profile (differentiating $\mathrm{AD}$ from DLB) [9].

Furthermore, using the quantitative $\mathrm{A} \beta$-immunoblot to analyze CSF samples of neuropathologically defined patients with $\mathrm{AD}$ (definite $\mathrm{AD}, \mathrm{dAD}$ ) and DLB (definite, $\mathrm{dDLB}$ ), the authors found that $A \beta(1-42 \%)$ was significantly lowered in $\mathrm{dAD}$ compared to nondemented controls (NDC) $(P=$ $\left.1.6 \times 10^{-7}\right)$. Oxidized $\mathrm{A} \beta(1-40 \mathrm{ox} \%)$ was elevated in $\mathrm{dDLB}$ as compared to $\operatorname{NDC}\left(P=1.8 \times 10^{-5}\right)$. Thus, it confirmed previous results on $A \beta$ peptide patterns in neuropathologically characterized patients with $\mathrm{AD}$ and DLB (Table 1 ). The results underscore the usefulness of CSF $A \beta(1-42 \%)$ and $\mathrm{A} \beta(1-40 \mathrm{ox} \%)$ as diagnostic biomarkers for $\mathrm{AD}$ and DLB, respectively $[118,119]$. As mentioned above, reduced GSH but not oxidized GSSH in CSF was significantly lower in Lewy body disease patients than control subjects [74]. To date, the implications of reduced GSH observed in $\mathrm{AD}$ patients are not yet clear (Table 1). However, differences in total glutathione concentrations in CSF for several neurodegenerative diseases including AD were not significant between groups [120], indicating that unlike reduced glutathione, the total glutathione is not useful as a CSF marker for assumed oxidative stress in patients with $\mathrm{PD}, \mathrm{AD}$, or multiple system atrophy.

Altered metal homeostasis may play a role in the pathogenesis of neurodegenerative disorders. It has been shown that patients with DLB had elevated Ca and Mg levels in CSF and Mg levels in plasma when compared to all other groups including $\mathrm{AD}$ and healthy controls $(P<0.001)$. A combination of CSF-Mg and CSF-Ca could distinguish DLB from AD with a sensitivity of $93 \%$ and a specificity of $85 \%$ [121]. Cu levels in both CSF and plasma tended to be higher in DLB compared to the other groups, but these trends failed to reach significance after correction for multiple comparisons. The observed elevations of CSF-Mg, CSF-Ca, and CSF-Cu may contribute to, or be associated with, the neurodegenerative process in DLB. Therefore, CSF$\mathrm{Mg}$ and CSF-Ca may be a valuable tool in distinguishing DLB from AD.

Neprilysin (NEP) is an A $\beta$-degrading protein found at presynaptic terminals and in body fluids. Reduced CSF NEP activity levels have been shown to occur in early AD; similarly, demented Lewy body disease patients had lowered CSF NEP activity levels, compared to both non-demented Lewy body disease subjects $(P=0.004)$ and controls $(P=0.02)$. In addition, CSF NEP activity levels correlated positively with CSF A $\beta 42$ levels which was not explained by the presence or absence of ApoE4 [122], suggesting that NEP is involved in both $\mathrm{AD}$ and DLB and altered CSF NEP activity may be a useful biomarker for dementia. Further, cystatin $\mathrm{C}(\mathrm{CysC})$ is a carrier of soluble $\mathrm{A} \beta 42$ in $\mathrm{CSF}$ and reduces $\mathrm{A} \beta$ plaque formation. Demented Lewy body disease patients had decreased CSF CysC levels. The correlation between CSF CysC and $\mathrm{A} \beta 42$ levels was high in non-demented subjects, 
TABLE 1: Differences of main CSF biomarkers in DLB and AD.

\begin{tabular}{|c|c|c|c|c|c|c|c|c|}
\hline & $\mathrm{A} \beta 42$ & T-tau & P-tau & $\alpha$ Syn & CART & $\mathrm{A} \beta 40 \mathrm{ox}$ & $\mathrm{F}_{2}$-IsoP & GSH \\
\hline Healthy & - & - & - & - & - & - & - & - \\
\hline DLB & - or $\downarrow$ & - & - & $\downarrow$ & $\downarrow \downarrow$ & $\uparrow$ & ? & $\downarrow$ \\
\hline $\mathrm{AD}$ & $\downarrow \downarrow$ & $\uparrow \uparrow$ & $\uparrow \uparrow$ & - & $\downarrow$ & - & $\uparrow$ & $?$ \\
\hline
\end{tabular}

-: normal level; $\downarrow$ : decrease; $\uparrow:$ increase; double arrows: full changes; ?: unknown.

but poor in demented patients, indicating that low CSF CysC levels are associated with dementia, possibly through a disturbed elimination of soluble $\mathrm{A} \beta 42$ [123].

Hyaluronic acid (HA) is an adhesion molecule known to regulate both vascular and inflammatory processes. Recent analyses showed that male AD and DLB patients had almost double the amount of HA compared to female patients whereas no gender differences were observed in the controls. Furthermore, CSF levels of HA in most female AD patients correlated with various AD-related biomarkers. Correlations between HA levels and markers of inflammation and vascular changes were only detected in female $\mathrm{AD}$ patients but in both male and female DLB patients. Therefore the HA profile in CSF, but not in plasma, and associations to other markers appear to be gender dependent which should be taken into account in clinical examinations and future biomarker studies [124].

Some hopeful DLB biomarkers in CSF are listed in Table 1, including neuropeptide CART discussed below.

\section{Neurotransmitter CART, a Potential Biomarker for DLB or Causative Factor for Dementia}

CART peptide is broadly expressed in the cortex, hippocampus, amygadala, hypothalamus, pituitary, and some endocrine glands $[125,126]$. This suggests a general role in different cells. Very recently, we found that CART has a general cytoprotection function in mammalian and human cells [11]. We found that CART protects mitochondrial DNA (mtDNA), cellular proteins, and lipids against the oxidative action of hydrogen peroxide. Using cis-parinaric acid as a sensitive reporting probe for peroxidation in membranes and a lipid-soluble azo initiator of peroxyl radicals, we found that CART has an antioxidant property. CART is preferentially localized in mitochondria, which are the main source of free radicals. Interestingly, this antioxidant and protective role is also seen in a mouse PD model [11]. Therefore we propose that CART is an important antioxidant hormone. These mitochondria associated cytoprotection and trophic roles are particularly important in the development of neurodegenerative diseases, including $\mathrm{AD}$ and $\mathrm{DLB}$, since most of them have a mitochondrial dysfunction or an energy failure condition.

MRI study has shown that patients with DLB displayed hypothalamic atrophy whereas this region was not affected in $\mathrm{AD}$ patients [127]. CART is a neuropeptide expressed selectively in neurons in the hypothalamus. It is, therefore, possible that neurons producing CART in the hypothalamus are affected in DLB. Although Lewy bodies have been described in the hypothalamus of DLB patients [128], extensive neuropathological studies of this region in DLB have not yet been reported.Consequently, it would be of interest to study whether neurons expressing CART are affected in DLB as well as analyzing the role of changed CSF-CART levels. Using sensitive and commercially available radioimmunoassay (RIA), Schultz et al. [129] found that CSF-CART levels were significantly reduced by $30 \%$ in DLB patients compared to controls as well as $\mathrm{AD}$ patients. These results suggest that reduced CSF CART is a sign of hypothalamic dysfunction in DLB and that it may serve as a new biomarker for DLB patients. A summary of CSF biomarkers for DLB is shown in Table 1.

Interestingly, CSF dopamine metabolite (DOPAC) level is correlated with behavioral and psychological signs and symptoms of dementia (BPSD) in general [130]. Intracerebroventricular injections of CART in rodents lead to increased dopaminergic activity in nucleus accumbens, striatum, and hypothalamus [131] and systemic injections of CART in a PD mouse model preserve dopaminergic neurons in SN area [11]. Reduced CART levels may, therefore, be involved in causing or augmenting the dopaminergic hypofunction resulting in Parkinsonism as well as other symptoms, including mood/psychiatric symptoms [132]. CART level is also reduced in AD patients [129]. The precise mechanisms of CART underlining these changes and the significance of CART in both DLB and AD diseases need further investigation in the future.

Although the involvement of CART in mitochondria and neurotrophin signaling in healthy and injured neurons is increasingly recognized, the impact of CART on the cellular processing of amyloid precursor protein tau and alphasynuclein, the key genes/proteins associated AD and DLB, is currently unexplored.

\section{The Differences between Central and Peripheral Biomarkers}

The utility of plasma $A \beta 42$ and other $A \beta$ peptides as biomarkers for $\mathrm{AD}$ is less compelling and concentrations of $\mathrm{A} \beta 42$ in cerebrospinal fluid and plasma show no apparent correlation $[69,133-138]$. However, since the blood test is noninvasive, easily applicable, and cost effective, peripheral biomarkers especially blood or serum biomarkers are definitely desirable, and some reports suggest that they may be promising biomarkers.

Alzheimer's dementia is a systemic or global metabolism disorder. Some important changes in the body or circulatory system may occur much earlier than clinical onset. Plasma 
$\mathrm{A} \beta$ levels are elevated in early-onset AD caused by autosomal dominant mutations. Notably, it has been reported that plasma $\mathrm{A} \beta 42$, but not $\mathrm{A} \beta 40$, is also significantly elevated in late-onset $\mathrm{AD}$ first degree, compared to controls, determined by using a specific monoclonal antibody against $\mathrm{A} \beta 42$ or A $\beta 40$ [139]. Similarly, higher plasma $A \beta 42$ at baseline was a significant predictor for the conversion to probable or possible $\mathrm{AD}$ at 5 years. Higher conversion to $\mathrm{AD}$ was also associated with male gender but not with either higher scores on the Geriatric Depression Scale, stroke, cerebral infarction, or apolipoprotein E $\varepsilon 4$ allele [140]. This A $\beta 42$ level may be changed in the progression of the disease prior to the development of overt dementia, due to the formation of $\mathrm{A} \beta$ plaques in the brain [114].

In contrast to CSF, plasma and urinary F2-IsoPs are not increased in probable AD patients [141]. In combination, these data indicate that CSF F2-IsoPs, but not peripheral F2IsoPs, are biomarkers of oxidative damage to the brain in AD. Since arachidonic acid is distributed among neuronal and nonneuronal elements in brain, F2-IsoPs are not specific for neuronal oxidative damage; they reflect a total oxidative damage (lipid peroxidation) in the brain $[112,142]$.

The AD brain is marked by severe neuronal death, which has been partly attributed to increased oxidative stress. The pathophysiology accounting for this free radical injury is not well delineated at this point but it is thought that a derangement in transition metal metabolism contributes to the process. Significantly decreased loosely bound iron in the hippocampal white matter of mild-moderate and severe $\mathrm{AD}$ patients and a trend towards increased nonheme iron in the hippocampal gray matter of severe $\mathrm{AD}$ patients have been observed. Furthermore, decreased levels of total copper were seen in severe $\mathrm{AD}$ and DLB frontal cortex compared to controls, suggesting an imbalance in brain metal levels in both $\mathrm{AD}$ and DLB. The decrease in loosely bound iron in mild-moderate $\mathrm{AD}$ patients may be associated with myelin breakdown seen in the beginning stages of $\mathrm{AD}$ and implies that iron dysregulation is an early event in $\mathrm{AD}$ pathogenesis [143].

Interestingly, peripheral derangement of transition metal metabolism is present early in the dementing process. An increase in the ratio of serum copper to nonheme iron levels predicted which subjects with mild cognitive impairment would progress to dementia versus those who would remain cognitively stable [144]. No gene was identified as being dysregulated more than 2-fold in a cDNA-based microarray (IronChip) containing genes relevant to iron and copper metabolism used to assess transition metal metabolism in circulating lymphocytes from cognitively normal and demented subjects. Therefore, the increased ratio of serum copper/iron prior to dementia has potential as a biomarker for cognitive decline and mirrors other changes in serum previously reported by others, but iron and copper metabolism pathways appear to be broadly unaffected in peripheral blood in $\mathrm{AD}$ [144].

Oxidative stress in dementia is not only a brain condition but also a global problem, and it may be monitored by urinary markers. 8 -Hydroxy- $2^{\prime}$-deoxyguanosine $(8-\mathrm{OHdG})$ is a biomarker indicating oxidative DNA damage. Paraoxonase 1 (PON1) is a high-density lipoprotein (HDL)-associated antioxidant enzyme and prevents oxidation of low-density lipoproteins. Urinary 8-OHdG levels were found to be significantly increased, but serum PON1 activity was significantly decreased in AD patients compared to controls [145]. Hence, oxidant stress and oxidative DNA damage are important pathological processes in $\mathrm{AD}$, and such oxidation related status of patients with $\mathrm{AD}$ might be easily determined and monitored by these biomarkers, urinary 8 -OHdG level and serum PON1 activity. These peripheral biomarkers totally reflect an imbalance of oxidant and antioxidant and the systemic condition of oxidative stress, supporting the view that mitochondrial dysfunction and oxidative stress may be the initial events prior to the amyloid accumulation (Figure 1). This mitochondria/oxidative stress hypothesis may apply mostly to the late onset of AD [146, 147].

Some neurotrophic factors in serum may be changed in dementia patients. Glial cell-line derived neurotrophic factor (GDNF) has been extensively studied for its neuroprotective role in vitro and in vivo. Straten et al. compared GDNF concentrations in CSF and serum of patients with AD and normal controls (NC). While GDNF concentrations in CSF were significantly increased in patients with $\mathrm{AD}$ (291.7 pg/mL) compared with NC subjects $(218.7 \mathrm{pg} / \mathrm{mL}, P=$ $0.012)$, GDNF concentrations of AD patients $(486.5 \mathrm{pg} / \mathrm{mL})$ in serum were significantly decreased compared with the NC group $(711.5 \mathrm{pg} / \mathrm{mL}, P<0.001)$. It was thought that increased GDNF in CSF of AD might be due to an upregulated expression in the CNS as an adaptive process of the impaired brain to enhance neurotrophic support at least in early stages of disease and/or impairment of CSF turnover [148]. It is curious that CSF GDNF increased but serum GDNF significantly decreased in AD patients.

A general and unbiased approach to the identification of diagnostically useful antibodies that avoids the requirement for antigen identification has been developed recently [149]. This method involves the comparative screening of combinatorial libraries of unnatural synthetic molecules against serum samples obtained from cases and controls. Molecules that retain far more $\operatorname{IgG}$ antibodies from the case samples than the controls are identified and subsequently tested as capture agents for diagnostically useful antibodies. Using this method, two candidate IgG biomarkers for AD have been identified; they are AD peptoids: ADP1- (or 3-) binding antibodies and ADP2-binding antibodies. AD blood samples contained higher levels of antibodies that bind to ADP2 as well as antibodies that bind to ADP1 and ADP3 than non-AD samples [149], indicating they may be strong biomarkers for the disease. Certainly, more research is needed to determine whether the peptoids ADP1-3 will be useful reagents for the clinical diagnosis of AD.

Taken together, oxidative stress in dementia is probably a global pathological feature, it can be monitored by some central and peripheral biomarkers. $\mathrm{A} \beta$ biomarkers are likely downstream markers of mitochondrial and metabolism dysfunction [146]. Compared to CSF biomarkers for dementia, peripheral biomarkers are relatively less accurate and less consistent, probably because dementia is mainly a brain 
disorder and the local pathological factors closely reflect the nature of the disease. Array-based methods and other sensitive approaches may provide new, useful biomarkers for quick and accurate diagnosis of dementia and more readily distinguish its subtypes.

\section{Conclusions}

Oxidative stress is significantly involved in the pathology of degenerative Alzheimer's dementia and dementia with Lewy bodies, even in the early stages of the diseases. This feature supports an oxidative stress hypothesis for dementia (Figure 1). Disease-related biomarkers (i.e., oxidative stress-related chemicals) can provide diagnostic, prognostic, and therapeutic targets and also may address some etiological questions and important problems in clinical and translational medical research.

Importantly, not only in classic $\mathrm{AD}$ patients but also in people with mild cognitive impairment, CSF A $\beta 42$ levels are significantly decreased when compared to healthy adults. This reflects abnormal amyloid metabolism, one of the most important changes in the brain, suggesting the formation of some tiny amyloid plaques in the hippocampus and the cortex. At the same time or later, total tau levels, particularly p-tau levels, are largely increased in AD patients comparing to healthy controls.

Patients with DLB show either normal levels of these core CSF biomarkers or slightly elevated T-tau and slightly decreased $\mathrm{A} \beta 42$, while P-tau levels are around normal or only slightly elevated or slightly decreased. This distinguished DLB from $\mathrm{AD}$ and can be the first key point of the differential diagnosis. Furthermore, $\alpha$ Syn is decreased in most DLB cases compared to healthy controls as well as $\mathrm{AD}$ patients. This is the second distinction that may assist in the differential diagnosis.

In addition, among other biomarker candidates, endogenous antioxidant CART is very promising since deficits in serotonergic and dopaminergic pathways seem more pronounced in DLB patients, and CART can modulate these pathways [132]. In particular, clinic practice shows CART level in CSF is significantly decreased in DLB patients compared to AD as well as healthy controls [129]. Combining CART with the core markers may further increase diagnostic accuracy (Table 1).

In conclusion, the combination of CART and the brain specific proteins t-tau, p-tau, $\mathrm{A} \beta 42$, and $\alpha$ Syn in CSF are associated with the clinical diagnosis of DLB and discriminate between $\mathrm{AD}$ and DLB with high diagnostic accuracy, suggesting this combination as a new potential biomarker panel for DLB. If this diagnosis panel is confirmed by new investigations, it may be a great advance in neurodegenerative disease diagnostics and treatment.

\section{Acknowledgments}

This project was supported by the American Heart Association (AHA award, Beginning Grant-in-Aid no. 0565527Z), the National Center for Research Resources, and the Office of Research Infrastructure Programs (ORIP) of the National Institutes of Health through Grant number RR000163.

\section{References}

[1] D. S. Knopman, S. T. DeKosky, J. L. Cummings et al., "Practice parameter: diagnosis of dementia (an evidence-based review): report of the quality standards subcommittee of the american academy of neurology," Neurology, vol. 56, no. 9, pp. 1143-1153, 2001.

[2] B. T. Hyman, C. H. Phelps, T. G. Beach et al., "National institute on aging-alzheimer's association guidelines for the neuropathologic assessment of Alzheimer's disease," Alzheimer's \& Dementia, vol. 8, no. 1, pp. 1-13, 2012.

[3] N. S. Schoonenboom, F. E. Reesink, N. A. Verwey et al., "Cerebrospinal fluid markers for differential dementia diagnosis in a large memory clinic cohort," Neurology, vol. 78, no. 1, pp. 47-54, 2012.

[4] M. T. Lin and M. F. Beal, "Mitochondrial dysfunction and oxidative stress in neurodegenerative diseases," Nature, vol. 443, no. 7113, pp. 787-795, 2006.

[5] L. J. Martin, "Mitochondrial and cell death nechanisms in neurodegenerative diseases," Pharmaceuticals (Basel), vol. 3, no. 4, pp. 839-915, 2010.

[6] P. Mao and P. H. Reddy, "Aging and amyloid beta-induced oxidative DNA damage and mitochondrial dysfunction in Alzheimer's disease: implications for early intervention and therapeutics," Biochimica et Biophysica Acta, vol. 1812, no. 11, pp. 1359-1370, 2011.

[7] I. G. McKeith, D. J. Burn, C. G. Ballard et al., "Dementia with Lewy bodies," Seminars in Clinical Neuropsychiatry, vol. 8, no. 1, pp. 46-57, 2003.

[8] K. A. Jellinger, "Formation and development of Lewy pathology: a critical update," Journal of Neurology, vol. 256, no. 3, supplement, pp. S270-S279, 2009.

[9] E. B. Mukaetova-Ladinska, R. Monteith, and E. K. Perry, "Cerebrospinal fluid biomarkers for Dementia with Lewy bodies," International Journal of Alzheimer's Disease, vol. 2010, Article ID 536538, 17 pages, 2010.

[10] P. Mao, A. Ardeshiri, R. Jacks, S. Yang, P. D. Hurn, and N. J. Alkayed, "Mitochondrial mechanism of neuroprotection by CART," European Journal of Neuroscience, vol. 26, no. 3, pp. 624-632, 2007.

[11] P. Mao, C. K. Meshul, P. Thuillier, N. R. Goldberg, and P. H. Reddy, "CART peptide is a potential endogenous antioxidant and preferentially localized in mitochondria," PLOS ONE, vol. 7, no. 1, article e29343, 2012.

[12] J. E. Selfridge, L. Eb, J. Lu, and R. H. Swerdlow, "Role of mitochondrial homeostasisand dynamics in Alzheimer's disease," Neurobiology of Disease. In press.

[13] M. A. Lovell and W. R. Markesbery, "Oxidatively modified RNA in mild cognitive impairment," Neurobiology of Disease, vol. 29, no. 2, pp. 169-175, 2008.

[14] A. Nunomura, G. Perry, G. Aliev et al., "Oxidative damage is the earliest event in Alzheimer disease," Journal of Neuropathology and Experimental Neurology, vol. 60, no. 8, pp. 759-767, 2001.

[15] D. Praticò, K. Uryu, S. Leight, J. Q. Trojanoswki, and V. M. Y. Lee, "Increased lipid peroxidation precedes amyloid plaque formation in an animal model of alzheimer amyloidosis," Journal of Neuroscience, vol. 21, no. 12, pp. 4183-4187, 2001. 
[16] L. Migliore, I. Fontana, F. Trippi et al., “Oxidative DNA damage in peripheral leukocytes of mild cognitive impairment and $\mathrm{AD}$ patients," Neurobiology of Aging, vol. 26, no. 5, pp. 567-573, 2005.

[17] R. Sultana, D. Boyd-Kimball, H. F. Poon et al., "Redox proteomics identification of oxidized proteins in Alzheimer's disease hippocampus and cerebellum: an approach to understand pathological and biochemical alterations in AD," Neurobiology of Aging, vol. 27, no. 11, pp. 1564-1576, 2006.

[18] P. Bermejo, S. Martín-Aragón, J. Benedí et al., "Differences of peripheral inflammatory markers between mild cognitive impairment and Alzheimer's disease," Immunology Letters, vol. 117, no. 2, pp. 198-202, 2008.

[19] L. L. Torres, N. B. Quaglio, G. T. de Souza et al., "Peripheral oxidative stress biomarkers in mild cognitive impairment and Alzheimer's disease," Journal of Alzheimer's Disease, vol. 26, no. 1, pp. 59-68, 2011.

[20] M. A. Lovell and W. R. Markesbery, "Oxidative damage in mild cognitive impairment and early Alzheimer's disease," Journal of Neuroscience Research, vol. 85, no. 14, pp. 3036-3040, 2007.

[21] L. M. Sayre, D. A. Zelasko, P. L. R. Harris, G. Perry, R. G. Salomon, and M. A. Smith, "4-Hydroxynonenal-derived advanced lipid peroxidation end products are increased in Alzheimer's disease," Journal of Neurochemistry, vol. 68, no. 5, pp. 2092-2097, 1997.

[22] R. Sultana, P. Mecocci, F. Mangialasche, R. Cecchetti, M. Baglioni, and D. A. Butterfield, "Increased protein and lipid oxidative damage in mitochondria isolated from lymphocytes from patients with alzheimer's disease: insights into the role of oxidative stress in alzheimer's disease and initial investigations into a potential biomarker for this dementing disorder," Journal of Alzheimer's Disease, vol. 24, no. 1, pp. 77-84, 2011.

[23] J. Laurén, D. A. Gimbel, H. B. Nygaard, J. W. Gilbert, and S. M. Strittmatter, "Cellular prion protein mediates impairment of synaptic plasticity by amyloid-beta oligomers," Nature, vol. 457, no. 7233, pp. 1128-1132, 2009.

[24] D. J. Kyle, E. Schaefer, G. Patton, and A. Beiser, "Low serum docosahexaenoic acid is a significant risk factor for Alzheimer's dementia," Lipids, vol. 34, no. 6, supplement, p. S245, 1999.

[25] M. Soderberg, C. Edlund, K. Kristensson, and G. Dallner, "Fatty acid composition of brain phospholipids in aging and in Alzheimer's disease," Lipids, vol. 26, no. 6, pp. 421-425, 1991.

[26] M. R. Prasad, M. A. Lovell, M. Yatin, H. Dhillon, and W. R. Markesbery, "Regional membrane phospholipid alterations in Alzheimer's disease," Neurochemical Research, vol. 23, no. 1, pp. 81-88, 1998.

[27] J. Nourooz-Zadeh, E. H. C. Liu, B. Yhlen, E. E. Änggård, and B. Halliwell, "F4-isoprostanes as specific marker of docosahexaenoic acid peroxidation in Alzheimer's disease," Journal of Neurochemistry, vol. 72, no. 2, pp. 734-740, 1999.

[28] T. J. Montine, M. D. Neely, J. F. Quinn et al., "Lipid peroxidation in aging brain and Alzheimer's disease," Free Radical Biology and Medicine, vol. 33, no. 5, pp. 620-626, 2002.

[29] D. Praticò, V. M. Y. Lee, J. Q. Trojanowski, J. Rokach, and G. A. Fitzgerald, "Increased F2-isoprostanes in Alzheimer's disease: evidence for enhanced lipid peroxidation in vivo," The FASEB Journal, vol. 12, no. 15, pp. 1777-1783, 1998.

[30] G. P. Lim, F. Calon, T. Morihara et al., "A diet enriched with the omega-3 fatty acid docosahexaenoic acid reduces amyloid burden in an aged Alzheimer mouse model," Journal of Neuroscience, vol. 25, no. 12, pp. 3032-3040, 2005.
[31] C. R. Hooijmans, C. E. E. M. Van der Zee, P. J. Dederen et al., "DHA and cholesterol containing diets influence Alzheimerlike pathology, cognition and cerebral vasculature in APPswe/PS1dE9 mice," Neurobiology of Disease, vol. 33, no. 3, pp. 482-498, 2009.

[32] D. S. Auld, T. J. Kornecook, S. Bastianetto, and R. Quirion, "Alzheimer's disease and the basal forebrain cholinergic system: relations to $\beta$-amyloid peptides, cognition, and treatment strategies," Progress in Neurobiology, vol. 68, no. 3, pp. 209-245, 2002.

[33] R. Schliebs and T. Arendt, "The significance of the cholinergic system in the brain during aging and in Alzheimer's disease," Journal of Neural Transmission, vol. 113, no. 11, pp. 1625-1644, 2006.

[34] M. A. Daulatzai, "Early stages of pathogenesis in memory impairment during normal senescence and Alzheimer's disease," Journal of Alzheimer's Disease, vol. 20, no. 2, pp. 355-367, 2010.

[35] E. Tamagno, M. Guglielmotto, M. Aragno et al., "Oxidative stress activates a positive feedback between the $\gamma$ - and $\beta$ secretase cleavages of the $\beta$-amyloid precursor protein," Journal of Neurochemistry, vol. 104, no. 3, pp. 683-695, 2008.

[36] C. Shen, Y. Chen, H. Liu et al., "Hydrogen peroxide promotes $\mathrm{A} \beta$ production through JNK-dependent activation of $\gamma$ secretase," The Journal of Biological Chemistry, vol. 283, no. 25, pp. 17721-17730, 2008.

[37] H. Zetterberg, E. Mortberg, L. Song et al., "Hypoxia due to cardiac arrest induces a time-dependent increase in serum amyloid beta levels in humans," PLoS ONE, vol. 6, no. 12, article e28263, 2011.

[38] D. S. Bredt, P. M. Hwang, C. E. Glatt, C. Lowenstein, R. R. Reed, and S. H. Snyder, "Cloned and expressed nitric oxide synthase structurally resembles cytochrome P-450 reductase," Nature, vol. 351, no. 6329, pp. 714-718, 1991.

[39] D. S. Bredt and S. H. Snyder, "Transient nitric oxide synthase neurons in embryonic cerebral cortical plate, sensory ganglia, and olfactory epithelium," Neuron, vol. 13, no. 2, pp. 301-313, 1994.

[40] S. A. Austin, A. V. Santhanam, and Z. S. Katusic, "Endothelial nitric oxide modulates expression and processing of amyloid precursor protein," Circulation Research, vol. 107, no. 12, pp. 1498-1502, 2010.

[41] S. Arlt, F. Schulze, M. Eichenlaub et al., "Asymmetrical dimethylarginine is increased in plasma and decreased in cerebrospinal fluid of patients with Alzheimer's disease," Dementia and Geriatric Cognitive Disorders, vol. 26, no. 1, pp. 58-64, 2008.

[42] N. V. Morgan, S. K. Westaway, J. E. V. Morton et al., "PLA2G6, encoding a phospholipase $\mathrm{A}_{2}$, is mutated in neurodegenerative disorders with high brain iron," Nature Genetics, vol. 38, no. 7, pp. 752-754, 2006.

[43] E. Grünblatt, J. Bartl, and P. Riederer, "The link between iron, metabolic syndrome, and Alzheimer's disease," Journal of Neural Transmission, vol. 118, no. 3, pp. 371-379, 2011.

[44] A. Mcneill and P. F. Chinnery, "Neurodegeneration with brain iron accumulation," Handbook of Clinical Neurology, vol. 100, pp. 161-172, 2011.

[45] M. A. Smith, P. L. R. Harris, L. M. Sayre, and G. Perry, "Iron accumulation in Alzheimer disease is a source of redoxgenerated free radicals," Proceedings of the National Academy of Sciences of the United States of America, vol. 94, no. 18, pp. 9866-9868, 1997. 
[46] M. A. Smith, X. Zhu, M. Tabaton et al., "Increased iron and free radical generation in preclinical Alzheimer disease and mild cognitive impairment," Journal of Alzheimer's Disease, vol. 19, no. 1, pp. 353-372, 2010.

[47] P. K. Mandal, M. Tripathi, and S. Sugunan, "Brain oxidative stress: detection and mapping of anti-oxidant marker "Glutathione" in different brain regions of healthy male/female, MCI and Alzheimer patients using non-invasive magnetic resonance spectroscopy," Biochemical and Biophysical Research Communications, vol. 417, no. 1, pp. 43-48, 2012.

[48] H. Vural, H. Demirin, Y. Kara, I. Eren, and N. Delibas, "Alterations of plasma magnesium, copper, zinc, iron and selenium concentrations and some related erythrocyte antioxidant enzyme activities in patients with Alzheimer's disease," Journal of Trace Elements in Medicine and Biology, vol. 24, no. 3, pp. 169-173, 2010.

[49] M. Y. Aksenov, H. M. Tucker, P. Nair et al., "The expression of key oxidative stress-handling genes in different brain regions in Alzheimer's disease," Journal of Molecular Neuroscience, vol. 11, no. 2, pp. 151-164, 1998.

[50] M. A. Lovell, C. Xie, and W. R. Markesbery, "Decreased glutathione transferase activity in brain and ventricular fluid in Alzheimer's disease," Neurology, vol. 51, no. 6, pp. 1562-1566, 1998.

[51] E. Barone, F. Di Domenico, G. Cenini et al., "Oxidative and nitrosative modifications of biliverdin reductase- $\mathrm{A}$ in the brain of subjects with Alzheimer's disease and amnestic mild cognitive impairment," Biochimica et Biophysica Acta, vol. 25, no. 4, pp. 623-633, 2011.

[52] E. Barone, C. Mancuso, F. Di Domenico et al., "Biliverdin reductase-A: a novel drug target for atorvastatin in a dog preclinical model of Alzheimer disease," Journal of Neurochemistry, vol. 120, no. 1, pp. 135-146, 2012.

[53] E. Barone, F. Di Domenico, R. Sultana et al., "Heme oxygenase1 posttranslational modifications in the brain of subjects with Alzheimer disease and mild cognitive impairment," Free Radical Biology \& Medicine, vol. 52, no. 11-12, pp. 2292-2301, 2012.

[54] R. A. Nixon and A. M. Cataldo, "The endosomal-lysosomal system of neurons: new roles," Trends in Neurosciences, vol. 18, no. 11, pp. 489-496, 1995.

[55] J. Smythies, "What is the function of receptor and membrane endocytosis at the postsynaptic neuron?" Proceedings of the Royal Society B, vol. 267, no. 1450, pp. 1363-1367, 2000.

[56] S. Grimm, A. Hoehn, K. J. Davies, and T. Grune, "Protein oxidative modifications in the ageing brain: consequence for the onset of neurodegenerative disease," Free Radical Research, vol. 45, no. 1, pp. 73-88, 2011.

[57] A. M. Cataldo, D. J. Hamilton, J. L. Barnett, P. A. Paskevich, and R. A. Nixon, "Abnormalities of the endosomal-lysosomal system in Alzheimer's disease: relationship to disease pathogenesis," Advances in Experimental Medicine and Biology, vol. 389, pp. 271-280, 1996.

[58] A. M. Cataldo, D. J. Hamilton, J. L. Barnett, P. A. Paskevich, and R. A. Nixon, "Properties of the endosomal-lysosomal system in the human central nervous system: disturbances mark most neurons in populations at risk to degenerate in Alzheimer's disease," Journal of Neuroscience, vol. 16, no. 1, pp. 186-199, 1996.

[59] A. M. Cataldo, J. L. Barnett, C. Pieroni, and R. A. Nixon, "Increased neuronal endocytosis and protease delivery to early endosomes in sporadic Alzheimer's disease: neuropathologic evidence for a mechanism of increased $\beta$-amyloidogenesis," Journal of Neuroscience, vol. 17, no. 16, pp. 6142-6151, 1997.

[60] T. Grune and K. J. Davies, "Breakdown of oxidized proteins as a part of secondary antioxidant defenses in mammalian cells," Biofactors, vol. 6, no. 2, pp. 165-172, 1997.

[61] P. Mao, M. Manczak, M. J. Calkins et al., "Mitochondriatargeted catalase reduces abnormal APP processing, amyloid beta production and BACE1 in a mouse model of Alzheimer's disease: implications for neuroprotection and lifespan extension," Human Molecular Genetics, vol. 21, no. 13, pp. 2973-2990, 2012.

[62] S. E. Schriner, N. J. Linford, G. M. Martin et al., "Medecine: extension of murine life span by overexpression of catalase targeted to mitochondria," Science, vol. 308, no. 5730, pp. 1909-1911, 2005.

[63] M. J. De Leon, S. Desanti, R. Zinkowski et al., "MRI and CSF studies in the early diagnosis of Alzheimer's disease," Journal of Internal Medicine, vol. 256, no. 3, pp. 205-223, 2004.

[64] M. Brys, L. Glodzik, L. Mosconi et al., "Magnetic resonance imaging improves cerebrospinal fluid biomarkers in the early detection of Alzheimer's disease," Journal of Alzheimer's Disease, vol. 16, no. 2, pp. 351-362, 2009.

[65] K. Blennow and H. Zetterberg, "Is it time for biomarkerbased diagnostic criteria for prodromal Alzheimer's disease?" Alzheimer's Research and Therapy, vol. 2, no. 2, article 8, 2010.

[66] G. M. McKhann, D. S. Knopman, H. Chertkow et al., "The diagnosis of dementia due to Alzheimer's disease: recommendations from the National Institute on Aging-Alzheimer's Association workgroups on diagnostic guidelines for Alzheimer's disease," Alzheimer's and Dementia, vol. 7, no. 3, pp. 263-269, 2011.

[67] D. Prvulovic and H. Hampel, "Amyloid $\beta(\mathrm{A} \beta)$ and phosphotau (p-tau) as diagnostic biomarkers in Alzheimer's disease," Clinical Chemistry and Laboratory Medicine, vol. 49, no. 3, pp. 367-374, 2011.

[68] S. Vos, I. van Rossum, L. Burns et al., "Test sequence of CSF and MRI biomarkers for prediction of AD in subjects with MCI," Neurobiology of Aging, vol. 33, no. 10, pp. 2272-2281, 2012.

[69] R. J. Perrin, A. M. Fagan, and D. M. Holtzman, "Multimodal techniques for diagnosis and prognosis of Alzheimer's disease," Nature, vol. 461, no. 7266, pp. 916-922, 2009.

[70] I. G. McKeith, D. Galasko, K. Kosaka et al., "Consensus guidelines for the clinical and pathologic diagnosis of dementia with Lewy bodies (DLB): report of the consortium on DLB international workshop," Neurology, vol. 47, no. 5, pp. 1113-1124, 1996.

[71] I. G. McKeith, D. W. Dickson, J. Lowe et al., "Diagnosis and management of dementia with Lewy bodies: third report of the DLB consortium," Neurology, vol. 65, no. 12, pp. 1863-1872, 2005.

[72] J. B. Leverenz, I. Umar, Q. Wang et al., "Proteomic identification of novel proteins in cortical Lewy bodies," Brain Pathology, vol. 17, no. 2, pp. 139-145, 2007.

[73] T. G. Beach, C. H. Adler, L. I. Sue et al., "Reduced striatal tyrosine hydroxylase in incidental Lewy body disease," Acta Neuropathologica, vol. 115, no. 4, pp. 445-451, 2008.

[74] W. Maetzler, S. P. Schmid, I. Wurster et al., "Reduced but not oxidized cerebrospinal fluid glutathione levels are lowered in Lewy body diseases," Movement Disorders, vol. 26, no. 1, pp. 176-181, 2011.

[75] L. Warr and Z. Walker, "Identification of biomarkers in Lewybody disorders," Quarterly Journal of Nuclear Medicine and Molecular Imaging, vol. 56, no. 1, pp. 39-54, 2012. 
[76] N. Sinha, M. Firbank, and J. T. O’Brien, "Biomarkers in dementia with Lewy bodies: a review," International Journal of Geriatric Psychiatry, vol. 27, no. 5, pp. 443-453, 2011.

[77] E. Dalfó, M. Portero-Otín, V. Ayala, A. Martínez, R. Pamplona, and I. Ferrer, "Evidence of oxidative stress in the neocortex in incidental Lewy body disease," Journal of Neuropathology and Experimental Neurology, vol. 64, no. 9, pp. 816-830, 2005.

[78] E. Dalfó and I. Ferrer, "Early $\alpha$-synuclein lipoxidation in neocortex in Lewy body diseases," Neurobiology of Aging, vol. 29, no. 3, pp. 408-417, 2008.

[79] A. Navarro, A. Boveris, M. J. Bández et al., "Human brain cortex: mitochondrial oxidative damage and adaptive response in Parkinson disease and in dementia with Lewy bodies," Free Radical Biology and Medicine, vol. 46, no. 12, pp. 1574-1580, 2009.

[80] P. Mao, P. Gallagher, S. Nedungadi et al., "Mitochondrial DNA deletions and differential mitochondrial DNA content in Rhesus monkeys: implications for aging," Biochimica et Biophysica Acta, vol. 1822, no. 2, pp. 111-119, 2012.

[81] K. Kume, M. Kikukawa, H. Hanyu et al., "Telomere length shortening in patients with dementia with Lewy bodies," European Journal of Neurology, vol. 19, no. 6, pp. 905-910, 2012.

[82] A. M. Valdes, T. Andrew, J. P. Gardner et al., "Obesity, cigarette smoking, and telomere length in women," The Lancet, vol. 366, no. 9486, pp. 662-664, 2005.

[83] J. M. J. Houben, H. J. J. Moonen, F. J. van Schooten, and G. J. Hageman, "Telomere length assessment: biomarker of chronic oxidative stress?” Free Radical Biology and Medicine, vol. 44, no. 3, pp. 235-246, 2008.

[84] M. Balasubramanyam, A. Adaikalakoteswari, Z. Sameermahmood, and V. Mohan, "Biomarkers of oxidative stress: methods and measures of oxidative DNA damage (COMET assay) and telomere shortening," Methods in Molecular Biology, vol. 610, pp. 245-261, 2010.

[85] S. Masi, K. D. Salpea, K. Li et al., "Oxidative stress, chronic inflammation, and telomere length in patients with periodontitis," Free Radical Biology and Medicine, vol. 50, no. 6, pp. 730-735, 2011.

[86] O. M. Wolkowitz, S. H. Mellon, E. S. Epel et al., "Leukocyte telomere length in major depression: correlations with chronicity, inflammation and oxidative stress-preliminary findings," PLoS ONE, vol. 6, no. 3, article e17837, 2011.

[87] K. D. Salpea, P. J. Talmud, J. A. Cooper et al., "Association of telomere length with type 2 diabetes, oxidative stress and UCP2 gene variation," Atherosclerosis, vol. 209, no. 1, pp. 42-50, 2010.

[88] A. M. Valdes, I. J. Deary, J. Gardner et al., "Leukocyte telomere length is associated with cognitive performance in healthy women," Neurobiology of Aging, vol. 31, no. 6, pp. 986-992, 2010.

[89] F. Grodstein, M. van Oijen, M. C. Irizarry et al., "Shorter telomeres may mark early risk of dementia: preliminary analysis of 62 participants from the Nurses' Health Study," PLoS ONE, vol. 3, no. 2, article e1590, 2008.

[90] A. Ahmad, C. S. Burns, A. L. Fink, and V. N. Uversky, "Peculiarities of copper binding to alpha-synuclein," Journal Biomolecular Structure and Dynamics, vol. 29, no. 4, pp. 825-842, 2012.

[91] A. Gómez and I. Ferrer, "Increased oxidation of certain glycolysis and energy metabolism enzymes in the frontal cortex in lewy body diseases," Journal of Neuroscience Research, vol. 87, no. 4, pp. 1002-1013, 2009.
[92] J. H. T. Power and P. C. Blumbergs, "Cellular glutathione peroxidase in human brain: cellular distribution, and its potential role in the degradation of Lewy bodies in Parkinson's disease and dementia with Lewy bodies," Acta Neuropathologica, vol. 117, no. 1, pp. 63-73, 2009.

[93] M. Manczak, P. Mao, M. J. Calkins et al., "Mitochondriatargeted antioxidants protect against amyloid- $\beta$ toxicity in Alzheimer's disease neurons," Journal of Alzheimer's Disease, vol. 20, no. 2, supplement, pp. S609-S631, 2010.

[94] K. Blennow, H. Hampel, M. Weiner, and H. Zetterberg, "Cerebrospinal fluid and plasma biomarkers in Alzheimer disease," Nature Reviews Neurology, vol. 6, no. 3, pp. 131-144, 2010.

[95] N. Andreasen, L. Minthon, A. Clarberg et al., "Sensitivity, specificity, and stability of CSF-tau in AD in a communitybased patient sample," Neurology, vol. 53, no. 7, pp. 1488-1494, 1999.

[96] H. Hampel, S. J. Teipel, T. Fuchsberger et al., "Value of CSF $\beta$-amyloid1-42 and tau as predictors of Alzheimer's disease in patients with mild cognitive impairment," Molecular Psychiatry, vol. 9, no. 7, pp. 705-710, 2004.

[97] O. Hansson, H. Zetterberg, P. Buchhave, E. Londos, K. Blennow, and L. Minthon, "Association between CSF biomarkers and incipient Alzheimer's disease in patients with mild cognitive impairment: a follow-up study," The Lancet Neurology, vol. 5, no. 3, pp. 228-234, 2006.

[98] A. M. Fagan, C. M. Roe, C. Xiong, M. A. Mintun, J. C. Morris, and D. M. Holtzman, "Cerebrospinal fluid tau/ $\beta$-amyloid42 ratio as a prediction of cognitive decline in nondemented older adults," Archives of Neurology, vol. 64, no. 3, pp. 343-349, 2007.

[99] P. Lewczuk, J. Kornhuber, H. Vanderstichele et al., "Multiplexed quantification of dementia biomarkers in the CSF of patients with early dementias and MCI: a multicenter study," Neurobiology of Aging, vol. 29, no. 6, pp. 812-818, 2008.

[100] N. Mattsson, H. Zetterberg, O. Hansson et al., "CSF biomarkers and incipient Alzheimer disease in patients with mild cognitive impairment," Journal of the American Medical Association, vol. 302, no. 4, pp. 385-393, 2009.

[101] L. M. Shaw, H. Vanderstichele, M. Knapik-Czajka et al., "Qualification of the analytical and clinical performance of CSF biomarker analyses in ADNI," Acta Neuropathologica, vol. 121, no. 5, pp. 597-609, 2011.

[102] D. R. Lachno, M. J. Romeo, E. R. Siemers et al., "Validation of ELISA methods for quantification of total tau and phosporylated-tau181 in human cerebrospinal fluid with measurement in specimens from two Alzheimer's disease studies," Journal of Alzheimer's Disease, vol. 26, no. 3, pp. 531-541, 2011.

[103] H. Vanderstichele, M. Bibl, S. Engelborghs et al., "Standardization of preanalytical aspects of cerebrospinal fluid biomarker testing for Alzheimer's disease diagnosis: a consensus paper from the Alzheimer's Biomarkers Standardization Initiative," Alzheimer's and Dementia, vol. 8, no. 1, pp. 65-73, 2012.

[104] J. H. Kang, H. Vanderstichele, J. Q. Trojanowski, and L. M. Shaw, "Simultaneous analysis of cerebrospinal fluid biomarkers using microsphere-based xMAP multiplex technology for early detection of Alzheimer's disease," Methods, vol. 56, no. 4, pp. 484-493, 2012.

[105] M. Wagner, S. Wolf, F. M. Reischies et al., "Biomarker validation of a cued recall memory deficit in prodromal Alzheimer disease," Neurology, vol. 78, no. 6, pp. 379-386, 2012.

[106] R. H. Swerdlow and G. A. Jicha, "Alzheimer disease: can the exam predict the pathology?" Neurology, vol. 78, no. 6, pp. 374-375, 2012. 
[107] N. Mattsson, H. Zetterberg, O. Hansson et al., "Age and diagnostic performance of Alzheimer disease CSF biomarkers," Neurology, vol. 78, no. 7, pp. 468-476, 2012.

[108] L. Glodzik-Sobanska, E. Pirraglia, M. Brys et al., "The effects of normal aging and ApoE genotype on the levels of CSF biomarkers for Alzheimer's disease," Neurobiology of Aging, vol. 30, no. 5, pp. 672-681, 2009.

[109] C. N. Bassett, M. D. Neely, K. R. Sidell, W. R. Markesbery, L. L. Swift, and T. J. Montine, "Cerebrospinal fluid lipoproteins are more vulnerable to oxidation in Alzheimer's disease and are neurotoxic when oxidized ex vivo," Lipids, vol. 34, no. 12, pp. 1273-1280, 1999.

[110] T. J. Montine, M. F. Beal, M. E. Cudkowicz et al., "Increased CSF F2-isoprostane concentration in probable AD," Neurology, vol. 52, no. 3, pp. 562-565, 1999.

[111] K. S. Montine, J. F. Quinn, J. Zhang et al., "Isoprostanes and related products of lipid peroxidation in neurodegenerative diseases," Chemistry and Physics of Lipids, vol. 128, no. 1-2, pp. 117-124, 2004.

[112] T. J. Montine, J. A. Kaye, K. S. Montine, L. McFarland, J. D. Morrow, and J. F. Quinn, "Cerebrospinal fluid A $\beta 42$, tau, and F2-isoprostane concentrations in patients with Alzheimer disease, other dementias, and in age-matched controls," Archives of Pathology and Laboratory Medicine, vol. 125, no. 4, pp. 510-512, 2001.

[113] T. J. Montine, E. R. Peskind, J. F. Quinn, A. M. Wilson, K. S. Montine, and D. Galasko, "Increased cerebrospinal fluid F2isoprostanes are associated with aging and latent Alzheimer's disease as identified by biomarkers," Neuromolecular Medicine, vol. 13, no. 1, pp. 37-43, 2011.

[114] J. M. Ringman, S. G. Younkin, D. Pratico et al., "Biochemical markers in persons with preclinical familial Alzheimer disease," Neurology, vol. 71, no. 2, pp. 85-92, 2008.

[115] L. Mosconi, L. Glodzik, R. Mistur et al., "Oxidative stress and amyloid-beta pathology in normal individuals with a maternal history of Alzheimer's," Biological Psychiatry, vol. 68, no. 10, pp. 913-921, 2010.

[116] C. M. Gao, A. Y. Yam, X. Wang et al., "A $\beta 40$ oligomers identified as a potential biomarker for the diagnosis of alzheimer's disease," PLoS ONE, vol. 5, no. 12, article e15725, 2010.

[117] C. Isobe, T. Abe, and Y. Terayama, "Increase in the oxidized/total coenzyme q-10 ratio in the cerebrospinal fluid of alzheimer's disease patients," Dementia and Geriatric Cognitive Disorders, vol. 28, no. 5, pp. 449-454, 2009.

[118] M. Bibl, B. Mollenhauer, P. Lewczuk et al., "Validation of amyloid- $\beta$ peptides in CSF diagnosis of neurodegenerative dementias," Molecular Psychiatry, vol. 12, no. 7, pp. 671-680, 2007.

[119] B. Mollenhauer, H. Esselmann, C. Trenkwalder et al., "CSF amyloid- $\beta$ peptides in neuropathologically diagnosed dementia with lewy bodies and alzheimer's disease," Journal of Alzheimer's Disease, vol. 24, no. 2, pp. 383-391, 2011.

[120] C. H. Konings, M. A. Kuiper, T. Teerlink, C. Mulder, P. Scheltens, and E. C. Wolters, "Normal cerebrospinal fluid glutathione concentrations in Parkinson's disease, Alzheimer's disease and multiple system atrophy," Journal of the Neurological Sciences, vol. 168, no. 2, pp. 112-115, 1999.

[121] F. Boström, O. Hansson, L. Gerhardsson et al., "CSF Mg and Ca as diagnostic markers for dementia with Lewy bodies," Neurobiology of Aging, vol. 30, no. 8, pp. 1265-1271, 2009.
[122] W. Maetzler, V. Stoycheva, B. Schmid et al., "Neprilysin activity in cerebrospinal fluid is associated with dementia and amyloid$\beta 42$ levels in lewy body disease," Journal of Alzheimer's Disease, vol. 22, no. 3, pp. 933-938, 2010.

[123] W. Maetzler, B. Schmid, M. Synofzik et al., “The CST3 BB genotype and low cystatin $\mathrm{C}$ cerebrospinal fluid levels are associated with dementia in lewy body disease," Journal of Alzheimer's Disease, vol. 19, no. 3, pp. 937-942, 2010.

[124] H. M. Nielsen, S. Palmqvist, L. Minthon, E. Londos, and M. Wennström, "Gender-dependent levels of hyaluronic Acid in cerebrospinal fluid of patients with neurodegenerative dementia," Current Alzheimer Research, vol. 9, no. 3, pp. 257-266, 2012.

[125] G. Rogge, D. Jones, G. W. Hubert, Y. Lin, and M. J. Kuhar, "CART peptides: regulators of body weight, reward and other functions," Nature Reviews Neuroscience, vol. 9, no. 10, pp. 747-758, 2008.

[126] M. A. Upadhya, K. T. Nakhate, D. M. Kokare, P. S. Singru, and N. K. Subhedar, "Cocaine- and amphetamine-regulated transcript peptide increases spatial learning and memory in rats," Life Sciences, vol. 88, no. 7-8, pp. 322-334, 2011.

[127] J. L. Whitwell, S. D. Weigand, M. M. Shiung et al., "Focal atrophy in dementia with Lewy bodies on MRI: a distinct pattern from alzheimer's disease," Brain, vol. 130, no. 3, pp. 708-719, 2007.

[128] Y. S. Piao, K. Wakabayashi, S. Hayashi, M. Yoshimoto, and H. Takahashi, "Aggregation of $\alpha$-synuclein/NACP in the neuronal and glial cells in diffuse Lewy body disease: a survey of six patients," Clinical Neuropathology, vol. 19, no. 4, pp. 163-169, 2000.

[129] K. Schultz, S. Wiehager, K. Nilsson et al., "Reduced CSF CART in dementia with Lewy bodies," Neuroscience Letters, vol. 453, no. 2, pp. 104-106, 2009.

[130] S. Engelborghs, E. Vloeberghs, N. Le Bastard et al., "The dopaminergic neurotransmitter system is associated with aggression and agitation in frontotemporal dementia," Neurochemistry International, vol. 52, no. 6, pp. 1052-1060, 2008.

[131] K. R. Shieh, "Effects of the cocaine- and amphetamine-regulated transcript peptide on the turnover of central dopaminergic neurons," Neuropharmacology, vol. 44, no. 7, pp. 940-948, 2003.

[132] P. Mao, "Potential antidepressant role of neurotransmitter CART: implications for mental disorders," Depression Research and Treatment, vol. 2011, Article ID 762139, 11 pages, 2011.

[133] K. Blennow, G. de Meyer, O. Hansson et al., "Evolution of $\mathrm{A} \beta 42$ and $\mathrm{A} \beta 40$ levels and $\mathrm{A} \beta 42 / \mathrm{A} \beta 40$ ratio in plasma during progression of Alzheimer's disease: a multicenter assessment," Journal of Nutrition, Health and Aging, vol. 13, no. 3, pp. 205-208, 2009.

[134] N. Le Bastard, L. Aerts, J. Leurs, W. Blomme, P. P. De Deyn, and S. Engelborghs, "No correlation between time-linked plasma and CSF A $\beta$ levels," Neurochemistry International, vol. 55, no. 8, pp. 820-825, 2009.

[135] E. Mossello, E. Ballini, A. M. Mello et al., "Biomarkers of alzheimer's disease: from central nervous system to periphery?" International Journal of Alzheimer's Disease, vol. 2011, Article ID 342980, 7 pages, 2011.

[136] O. Hansson, H. Zetterberg, E. Vanmechelen et al., "Evaluation of plasma $A \beta 40$ and $A \beta 42$ as predictors of conversion to Alzheimer's disease in patients with mild cognitive impairment," Neurobiology of Aging, vol. 31, no. 3, pp. 357-367, 2010.

[137] M. Fei, W. Jianghua, M. Rujuan, Z. Wei, and W. Qian, "The relationship of plasma $\mathrm{A} \beta$ levels to dementia in aging individuals with mild cognitive impairment," Journal of the Neurological Sciences, vol. 305, no. 1-2, pp. 92-96, 2011. 
[138] F. Di Domenico, R. Coccia, D. A. Butterfield, and M. Perluigi, "Circulating biomarkers of protein oxidation for Alzheimer disease: expectations within limits," Biochimica et Biophysica Acta, vol. 1814, no. 12, pp. 1785-1795, 2011.

[139] N. Ertekin-Taner, L. H. Younkin, D. M. Yager et al., "Plasma amyloid beta protein is elevated in late-onset alzheimer disease families," Neurology, vol. 70, no. 8, pp. 596-606, 2008.

[140] I. Blasko, G. Kemmler, S. Jungwirth et al., "Plasma amyloid beta-42 independently predicts both late-onset depression and Alzheimer disease," American Journal of Geriatric Psychiatry, vol. 18, no. 11, pp. 973-982, 2010.

[141] T. J. Montine, L. Shinobu, K. S. Montine et al., "No difference in plasma or urinary F2-isoprostanes among patients with Huntington's disease or Alzheimer's disease and controls," Annals of Neurology, vol. 48, no. 6, p. 950, 2000.

[142] T. J. Montine, J. F. Quinn, K. S. Montine, J. A. Kaye, and J. C. S. Breitner, "Quantitative in vivo biomarkers of oxidative damage and their application to the diagnosis and management of Alzheimer's disease," Journal of Alzheimer's Disease, vol. 8, no. 4, pp. 359-367, 2005.

[143] S. Magaki, R. Raghavan, C. Mueller, K. C. Oberg, H. V. Vinters, and W. M. Kirsch, "Iron, copper, and iron regulatory protein 2 in Alzheimer's disease and related dementias," Neuroscience Letters, vol. 418, no. 1, pp. 72-76, 2007.

[144] C. Mueller, M. Schrag, A. Crofton et al., "Altered serum iron and copper homeostasis predicts cognitive decline in mild cognitive impairment," Journal of Alzheimer's Disease, vol. 29, no. 2, pp. 341-350, 2012.

[145] O. Zengi, A. Karakas, U. Ergun, M. Senes, L. Inan, and D. Yucel, "Urinary 8-hydroxy-2' -deoxyguanosine level and plasma paraoxonase 1 activity with Alzheimer's disease," Clinical Chemistry Laboratory Medicine, vol. 50, no. 3, pp. 529-534, 2011.

[146] R. H. Swerdlow, “Brain aging, Alzheimer's disease, and mitochondria," Biochimica et Biophysica Acta, vol. 1812, no. 12, pp. 1630-1639, 2011.

[147] R. H. Swerdlow, "Alzheimer's disease pathologic cascades: who comes first, what drives what," Neurotoxicity Research, vol. 22, no. 3, pp. 182-194, 2012.

[148] G. Straten, G. W. Eschweiler, W. Maetzler, C. Laske, and T. Leyhe, "Glial cell-line derived neurotrophic factor (GDNF) concentrations in cerebrospinal fluid and serum of patients with early Alzheimer's disease and normal controls," Journal of Alzheimer's Disease, vol. 18, no. 2, pp. 331-337, 2009.

[149] M. M. Reddy, R. Wilson, J. Wilson et al., "Identification of candidate IgG biomarkers for Alzheimer's disease via combinatorial library screening," Cell, vol. 144, no. 1, pp. 132-142, 2011. 


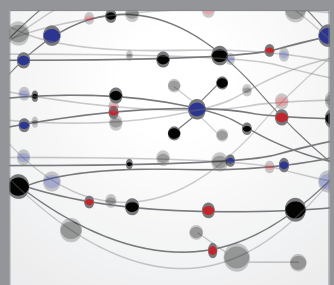

The Scientific World Journal
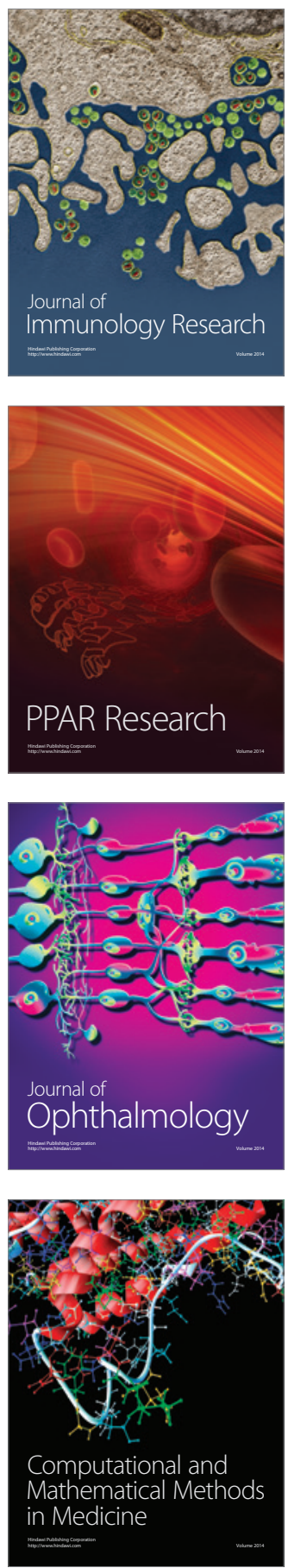

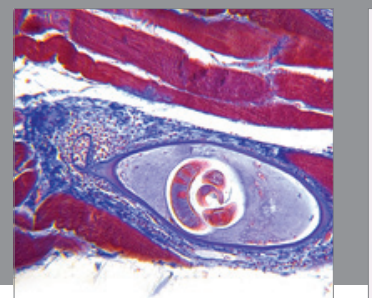

Gastroenterology

Research and Practice
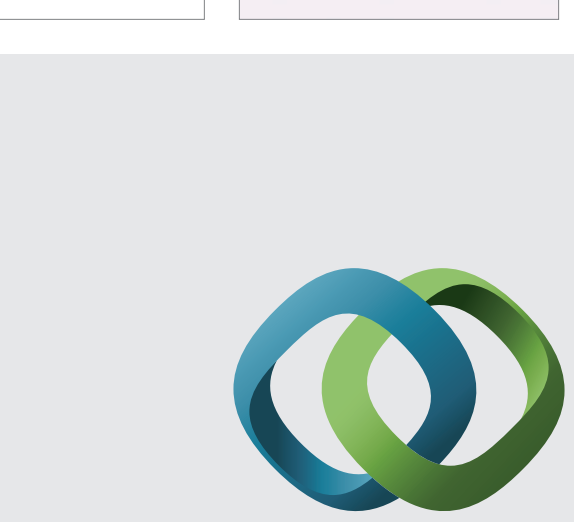

\section{Hindawi}

Submit your manuscripts at

http://www.hindawi.com
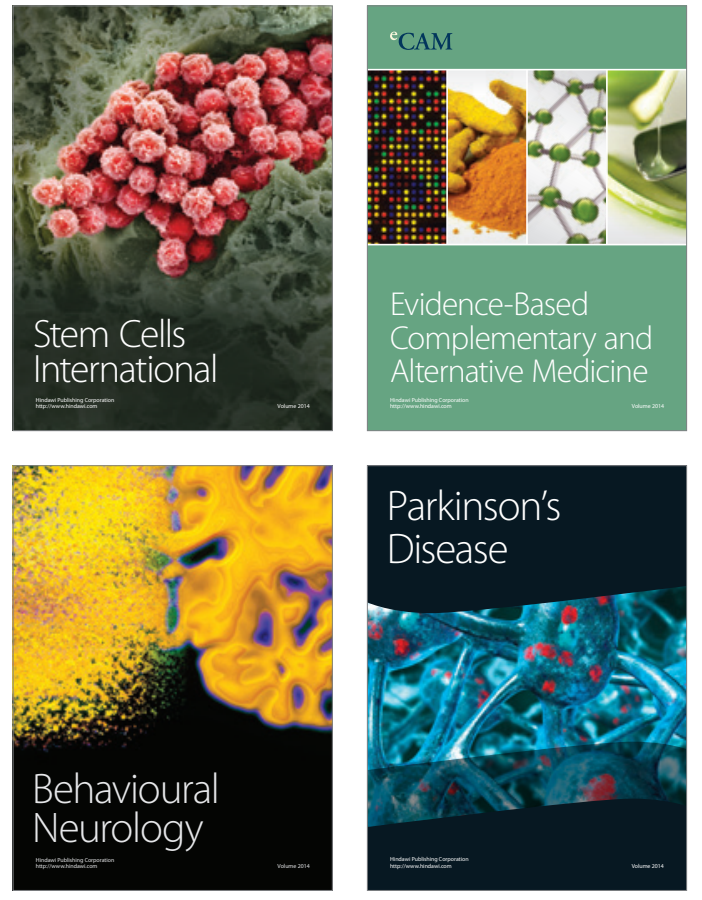
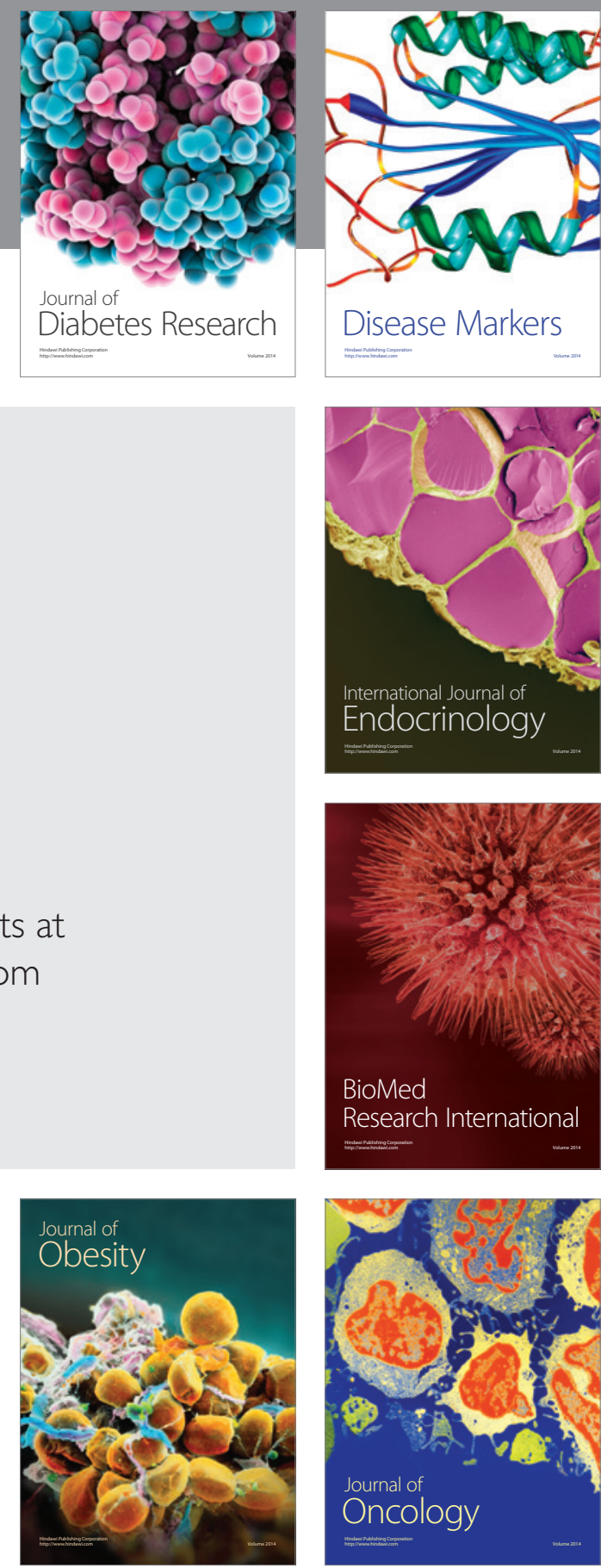

Disease Markers
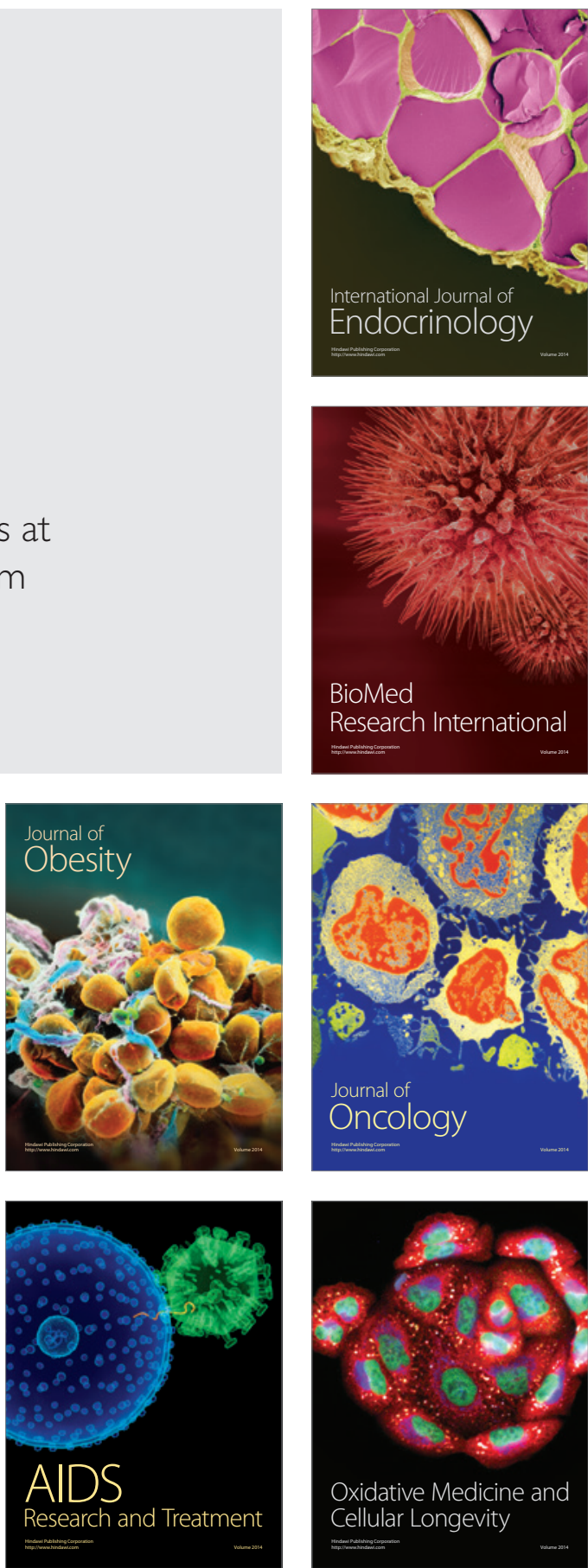